TFEC-2020-32505

\title{
Theoretical Investigation of Thermal Conductivity and Viscosity of Nanofluids
}

\author{
Alberto Soto1, Tyler Gaster1, Chris Golden1, Saeid Vafaei1* \\ 1Bradley University, 1501 W Bradley Ave, Peoria, IL 61625
}

\begin{abstract}
Working fluids are used every day in a plethora of applications ranging from electronics, automotive, aerospace and many more. With increasing demands for more efficient design and operation in electrical and mechanical components the criteria for cooling has consequently increased. We are at a point in design optimization where the thermal conductivity of working fluids has become a limiting factor in the design process. Cooling systems are imperative to the functionality of these components which is why it has become a modern challenge to manufacture working fluids with higher thermal conductivities, this is where nanofluids become a major point of interest. A nanofluid is a mixture of nanoparticles within a base fluid. Nanoparticles change the physical properties of the working fluids including the thermal conductivity and viscosity. This research was focused on expressing the effects of concentration and characteristics of nanoparticles including size and material on the nanofluids thermal conductivity and viscosity. It was also explained different thermal conductivity theoretical models that fall into three categories. These categories are the Effective Medium, Nanolayer and Brownian motion approaches for approximating nanofluid thermal conductivity. As well as theoretical models approximating viscosity of nanofluids. The level of accuracy of existing theoretical models was also examined through comparison to experimental data by calculating the average percent error. Observations have been made on the impact of temperature, concentration and size on viscosity and thermal conductivity. Generally, as the concentration was increased, the viscosity and thermal conductivity both increased. This research was focused to explain how to minimize the negative side effects of nanoparticles on viscosity of nanofluid. Indeed, the temperature can be raised to effectively reduce the viscosity while retaining the enhanced thermal conductivity of the nanofluid.
\end{abstract}

KEY WORDS: Thermal Conductivity, Effective Medium Theory, Nanolayer Theory, Agglomeration, Nanofluid

\section{INTRODUCTION}

As electronic devices become smaller and more powerful, transferring heat away from these devices becomes increasingly important. Stronger and more efficient electronics and mechanical power sources tend to generate more heat. Therefore, they require a coolant with the capacity to sufficiently remove heat to keep the system at optimal operating conditions. Design of modern electronics and efficient machinery is reaching a point where cooling capacity has become a limiting factor in the engineering process. This is why there is a peak interest in modifying the physical characteristics of working fluids in order to optimize systems.

One way to enhance the thermal management is to improve the physical properties of the working fluid, by addition of nanoparticles. The mixture of nanoparticles added to a base liquid are called nanofluids. Nanofluids can be used to improve the cooling system of devices from micro to macro scales. For example, nanoparticles can be added to the coolant of engines, thus increasing the cooling rates and lowering the size and weight of the radiator. This can lead to an increase in performance and efficiency of the engine [1].

*Corresponding Author: svafaei@bradley.edu 
Most working fluids have low thermal conductivity, the addition of nanoparticles having high thermal conductivity, most of the time modifies the overall thermal conductivity of nanofluid mixture and consequently, enhances the forced convection heat transfer. In general, the effects of nanoparticles on the thermal conductivity of nanofluids have been studied, experimentally and theoretically. There are several models to predict the thermal conductivity of nanofluids. The prediction of thermal conductivity of nanofluids started from simple models consisting of few rudimentary characteristics with low accuracy and developed to more sophisticated, more descriptive models with higher accuracy. The level of accuracy of theoretical models was examined by comparison to experimental data. Currently there has been no definitive consensus to what the driving mechanism is for the increase in thermal conductivities of nanofluids. Most researches compare their theoretical models with their own experimental data; however, it is imperative to compare different theoretical models with independent sources of experimental data in order to prevent any inherent biases.

The main objective in this investigation is (a) to examine the level of accuracy of different theoretical models by comparing the theoretical models against independent experimental data, (b) to suggest the most suitable theoretical model for given condition, by comparing the absolute average error of different models for the given experimental data. The existing theoretical equations are explained and compared against multiple sets of different experimental data to calculate an absolute average error percentage

\section{THEORETICAL MODELLING OF THERMAL CONCUDCTIVITY OF NANOFLUIDS}

In this section, different theoretical models are explained individually. The theoretical basis from which the models are derived will be explained in three separate sections. These sections are: Effective Medium Theory, Layering Model, and Brownian Motion. The corresponding models will be categorized within the sections they are associated with. The models are listed in order of complexity with Effective Medium Theory being the most traditional and rudimentary form of analysis, building up to Brownian motion being the most complex.

\subsection{Effective Medium Theory}

Effective Medium Theory approximation, also known as EMT, is an analytical approach used to predict the effective properties of a fluid mixture based on volume fraction of solute and properties of materials. EMT models are classified as static mechanisms since the theoretical model is based on stationary dispersion of solid nanoparticles throughout the base fluid as shown in Fig 1.

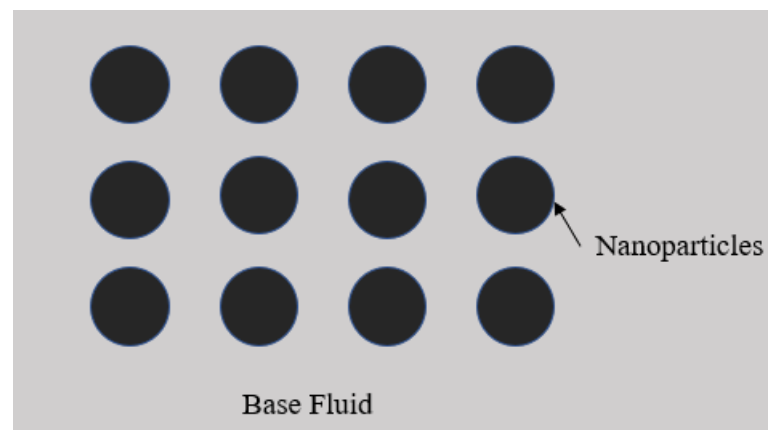

Fig. 1 A visual representation of the classical Effective Medium Theory

Maxwell [2] was the first to develop the static based theory in 1873, using micro and larger sized particles. Since then his model has been used as the foundation and baseline for all modern models improving accuracy with developing theories [2]. The Maxwell model [2] is described as the following equation 


$$
k_{n f}=\frac{k_{p}+2 k_{b f}+2 \phi\left(k_{p}-k_{b f}\right)}{k_{p}+2 k_{b f}-\phi\left(k_{p}-k_{b f}\right)} k_{b f}
$$

Where $k_{p}, k_{b f}$, and $k_{n f}$ are the thermal conductivity of nanoparticle, base fluid and nanofluid respectively. $\phi$ is the volume fraction concentration of the nanoparticle within the base fluid. It was reported that suspended particles ranging from the micrometre to millimetre levels can be considered in this model. In very dilute suspension mixtures of particle spheres, the interactions among particles were disregarded. The models were based on stationary dispersion of solid nanoparticles in a base fluid, representing a static model. The use of micromillimeter and millimetre sized particles in the solution resulted in clogging of micro channels of heat exchangers. As well as poor particle dispersion due to the large particles rapidly settling together and low conductivity enhancement at low particle concentration. As technology advanced the capability of using nanoparticles strongly impacted thermal conductivity of mixtures. Nanofluids are able to maintain a stable dispersion of particles throughout the fluid as well as increasing surface to volume ratio by a factor of 1000, producing a large enhancement of thermal conductivity at low concentrations. Further work on simple particle interactions was investigated by Hamilton Crosser [3], who proposed the thermal conductivity of a heterogeneous two component mixture also depended on the shape of the agglomerated nanoparticles. When nanoparticles are introduced into a fluid, they tend to agglomerate due to the cohesive interactions between high surface area particles. Hamilton Crosser [3] suggested that the thermal conductivity of the nanofluid is also dependent on the shape of these agglomerations, whether they be cylindrical or spherical, introducing

$$
k_{n f}=k_{b f}\left[\frac{k_{p}+(n-1) k_{b f}-(n-1)\left(k_{b f}-k_{p}\right) \phi}{k_{p}+(n-1) k_{b f}+\left(k_{b f}-k_{p}\right) \phi}\right]
$$

Where $n$ is the empirical shape factor representing the shape of agglomerated nanoparticles suspended within the base fluid. $n$ is either 3 for spherical particles or 6 for cylindrical particles, this physical characteristic is usually observed through the use of high-powered TEM microscopes. Wasp et al. [4] also developed a similar equation that predicts results similar to those of the Hamilton Crosser [3] under spherical particle conditions represented as

$$
k_{n f}=k_{b f} \frac{k_{n p}+2 k_{b f}-2 \phi\left(k_{b f}-k_{n p}\right)}{k_{n p}+2 k_{b f}+\phi\left(k_{b f}-k_{n p}\right)}
$$

Sundar et al [5] focused on monitoring the effect of thermal conductivity with the influence of temperature. Sundar et al [5] conducted his own experimental data in order to analyse the effect of the varying temperature. A correlation was proposed to formulate his experimental data-based equation shown as

$$
k_{n f}=k_{b f}(1+10.5 \phi)^{.1051}
$$

Under a specific range of temperature and nanoparticle concentration where $0<\phi<2.0 \%, 20^{\circ} \mathrm{C}<\mathrm{T}<60^{\circ} \mathrm{C}$. With an average deviation of $3.5 \%$ and standard deviation of $4.2 \%$, Sundar et al [40] was successfully able to better model an EMT based equation that can also predict the thermal conductivity of nanofluid with respect to temperature under volume fraction loadings and temperature within the given range. This is done by substituting the values of the thermal conductivities of the base fluid at the given temperatures.

\subsection{Layering Method}

One of the major mechanisms behind the enhancements when using nanofluids, is proposed to be the layering of liquid molecules at the solid-particle surface. The layer is an ordered liquid structure, surrounding the nanoparticle 
due to the interactions between the liquid and solid nanoparticle. This layering of liquid molecules is commonly referred to as the interfacial layer or nanolayer.

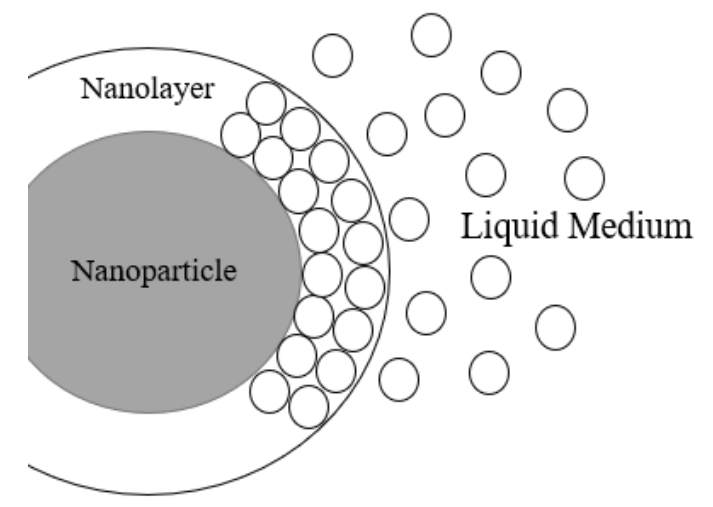

Fig 2. An illustration depicting the layering effect of a liquid medium on a nanoparticle. The nanoparticle is coloured in grey, and the surrounding base liquid molecules are shown adhering to the surface of the nanoparticle in an orderly fashion, causing a dense layer around the particle. The outer concentric circle surrounding the nanoparticle depict the formed nanolayer opposed to the surrounding liquid medium.

The nanoparticles atomic structures are more ordered than the atomic structures of the bulk liquid, making the nanolayer slightly denser than the base fluid. Since this interfacial layer is in an ordered solid-like state, it has a thermal conductivity greater than the bulk liquid and less than that of the solid particle. This solid-like layer acts as a thermal bridge between a solid nanoparticle and the base liquid closing the gap between thermal conductivities and increasing the overall heat transfer of the liquid mixture.

Xie et al [5] looked at the interfacial structures formed by liquid molecule layering at the nanoparticles surface and suggested with the assumption of a statistically homogenous and isotropic nanolayer that

$$
\frac{k_{n f}-k_{b f}}{k_{b f}}=3 \Theta \phi+\frac{3 \Theta^{2} \phi^{2}}{1-\Theta \phi}
$$

where $\Theta$ is defined by,

$$
\Theta=\frac{\left(\frac{k_{n l}-k_{b f}}{k_{n l}+2 k_{b f}}\right)\left[\left(1+\frac{\delta_{n l}}{r_{p}}\right)^{3}-\left[\frac{\left(k_{p}-k_{n l}\right)\left(k_{b f}+2 k_{n l}\right)}{\left(k_{p}+2 k_{n l}\right)\left(k_{b f}-k_{n l}\right)}\right]\right]}{\left(1+\frac{\delta_{n l}}{r_{p}}\right)^{3}+2\left[\left(\frac{k_{n l}-k_{b f}}{k_{n l}+2 k_{b f}}\right)\left(\frac{k_{p}-k_{n l}}{k_{p}+2 k_{n l}}\right)\right]}
$$

Where $k_{n l}$ is the thermal conductivity of the nanolayer, $r_{p}$ is the radius of nanoparticle, and $\delta_{n l}$ is the thickness of the nanolayer. The results from the developed equation indicate that the model is applicable to predict the effective thermal conductivities of various mixture solutions. It was assumed that the mixture system and the temperature fields within nanoparticle, nanolayer, and fluid were governed by steady state heat conduction. The physicochemical properties of the nanolayer are highly dependent on the: suspended nanoparticles, the base fluid, and the interactions between them. Currently there is no expressions for calculating the thermal conductivity of the layer due to the complexities behind the conditions of the layer and variables associated with them. Currently the nanolayer thermal conductivity is usually assumed to be a multiple of the base fluid thermal conductivity (generally 2 or 3). The created equation is based off the general heat conduction equation in spherical coordinates in addition to the equivalent hard sphere fluid model. The major assumption made in Xie's [5] equation is that the thermal conductivity of the nanolayer has a linear distribution. When the inclusion changes from a thermal 
insulation material to a high thermally conductive material, the thermal conductivity enhancement of the mixture increases, inversely. For improvement of the model a consideration of factors such as inclusion shape and surface chemistry should be considered. The effective thermal conductivity increases with a decrease of particle size and an increase in nanolayer thickness. With small particle sizes, the surface area is increased drastically resulting in greater interfacial layer's thickness. Leading to a more apparent nano-layer structure, implying that there is a correlation of the nanolayer and the particle size.

Considering the effect of volume fraction, thickness, thermal conductivity of the interfacial layer and particle size, and interfacial layer effect, a correlation was developed by Leong et al. [6], as

$$
k_{n f}=\frac{\left(k_{p}-k_{n l}\right) \phi k_{n l}\left[2\left(\beta_{n l}\right)^{3}-(\beta)^{3}+1\right]+\left(k_{p}+2 k_{n l}\right)\left(\beta_{n l}\right)^{3}\left[\phi(\beta)^{3}\left(k_{n l}-k_{b f}\right)+k_{b f}\right.}{\left(\beta_{n l}\right)^{3}\left(k_{p}+2 k_{n l}\right)-\left(k_{p}-k_{n l}\right) \phi\left[\left(\beta_{n l}\right)^{3}+(\beta)^{3}-1\right]}
$$

Where $\beta_{n l}$ and $\beta$ are dimensionless nanolayer constants calculated as

$$
\begin{gathered}
\beta_{n l}=1+\frac{\gamma}{2}, \\
\beta=1+\gamma
\end{gathered}
$$

Where $\gamma$ is the ratio of interfacial layer thickness to the nanoparticle radius:

$$
\gamma=\frac{\delta n l}{r_{p}}
$$

This developed model is used to determine the effective thermal conductivity of the nanofluid through the characterization of the particle size and nanolayer effect at the particle-liquid interface. If the nanolayer thickness diminishes or there is none, equation 7 reduces down the original Maxwell [2] equation. The interfacial layer has different thermo-physical properties for the bulk liquid and the solid particle, making it a separate component in the mixture. Their approach broke up the thermal conductivity into two separate parts, including 1) determination of the temperature fields and gradients of temperature, and 2) modelling of the effective thermal conductivity. The model for the thermal conductivity of the nanoparticle is comprised of three sections: the thermal conductivity of the nanoparticle itself, the thermal conductivity of the interfacial layer, and the thermal conductivity of the base liquid. The nanoparticles are all spherical and are far enough apart so there are no interactions in between particles, and the temperature fields are all continuous in all three components.

Tinga et al [13] had concluded a model taking into the account a complex dielectric constant of a multi-phase mixture in 1973. The multi phases being air-water-cellulose with confocal ellipsoidal shell. This model has been simplified in order to calculate a thermal conductivity instead of a dielectric constant by assuming the cellulose as solid particle, water as the interfacial layer and air as the host medium. The model is known as

$$
\frac{k_{n f}}{k_{b f}}=\left(1+\frac{3 \phi\left[\left(\beta^{3}-1\right)\left(2 k_{n l}+k_{p}\right)\left(k_{n l}-k_{b f}\right)-\left(k_{n l}-k_{p}\right)\left(2 k_{n l}+k_{b f}\right)\right]}{\left(2 k_{b f}+k_{n l}\right)\left(2 k_{n l}+k_{p}\right)-\frac{2}{\beta^{3}-1}\left(k_{n l}-k_{b f}\right)\left(k_{n l}-k_{p}\right)-3 \phi k_{n l}\left(k_{p}-k_{b f}\right)}\right)
$$

Yu and Choi [8] modified the Maxwell equation to include the effect of an ordered nanolayer as

$$
k_{n f}=\frac{k_{p}+2 k_{b f}+2\left(k_{p}-k_{b f}\right)(1-\gamma)^{3} \phi}{k_{p}+2 k_{b f}-\left(k_{p}-k_{b f}\right)(1+\gamma)^{3} \phi} k_{b f}
$$


They proposed that a solid-like nanolayer acts as a thermal path to close the gap between the solid nanoparticle and the surrounding bulk liquid conductivities. This nanolayer is theorized to have a significant role to the thermal conductivity of nanofluids. The solid-like nanolayer of the liquid molecules is assumed to have higher thermal conductivity than the base liquid, which is added to the traditional Maxwell equation. It is assumed that the thermal energy transport in the nanofluid is diffusive, which is justified due to the average interparticle distance in the nanofluid being significantly larger than the mean free path of the liquid molecules. Another significant assumption made was that the nanolayer around the particle is combined with the particle to form an equivalent particle, and that the particle volume concentration is so low that there is no overlap between the equivalent particles. For larger particles, the nanolayer impact is relatively smaller and the equation reduces to the original Maxwell equation [2].

\subsection{Brownian Model}

Brownian theory-based models take into account the random movement of particles in a fluid as a result of continuous collisions from molecules of the surrounding medium, enhancing the thermal conductivity of nanofluids. Localized convection can occur in the liquid due to the Brownian movement of the particles as well.

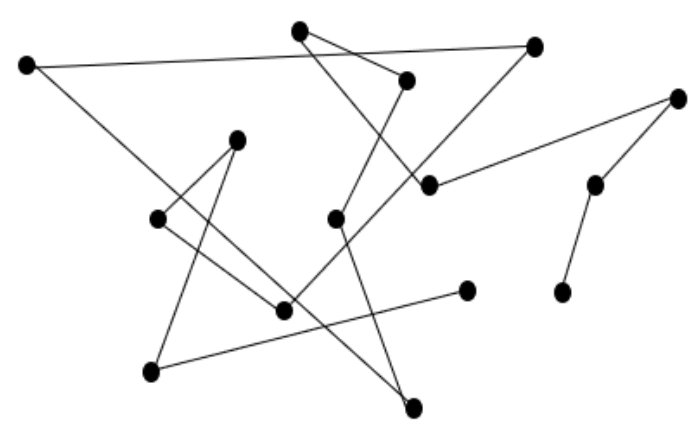

Fig. 4 An illustration of the random motion of a singular nanoparticle caused by Brownian motion.

Prasher et al [9] calculated the thermal conduction caused by Brownian movement and convection and proposed following expression

$$
k_{n f}=\left[\left(1+A \operatorname{Re}^{M} \operatorname{Pr}^{0.333} \phi\right)\left[\frac{\left(k_{p}(1+2 B i)+2 k_{m}\right)+2 \phi\left(k_{p}(1-B i)-k_{m}\right)}{\left(k_{p}(1+2 B i)+2 k_{m}\right)-\phi\left(k_{p}(1-B i)-k_{m}\right)}\right]\right] k_{b f}
$$

Where

$$
R e=\frac{1}{v} \sqrt{\frac{18 k_{b} T}{\pi \rho d}}
$$

As the particle size increases, Re or the Reynolds number approaches zero in this case the equation should reduce to the traditional Maxwell model [2]. $v, k_{b}, \rho, \mathrm{d}$, and $\mathrm{T}$ are: the kinematic viscosity of the liquid, Boltzmann constant, density, diameter of the nanoparticle, and temperature respectively. A, Pr and Bi are an empirical constant, the Prandtl number of the base fluid and Biot number of the nanoparticle respectively. $k_{m}$ is the matrix conductivity which is due to the convection by the movement of a single sphere. $k_{m}$ is calculated as 


$$
k_{m}=k_{b f}\left[1+\frac{1}{4 R e} \operatorname{Pr}\right]
$$

The Biot number of the nanoparticle is calculated

$$
B i=\frac{2 R_{b} k_{m}}{d_{n p}}
$$

Where $R_{b}$ is the thermal boundary resistance between nanoparticles and different fluids. Prasher et al [9] explained that there is more than just conduction to be considered to predict the thermal conductivity of nanofluids. Prasher [9] believes that Brownian movement as well as convection must also be considered in order to not underpredict thermal conductivity. They considered several mechanisms for thermal energy transfer in nanofluids: translation Brownian motion, existence of an interparticle potential, and convection in the liquid due to the Brownian movement. Analysis showed that the local convection due to this Brownian Motion was the most significant to effecting thermal conduction. The impact of interfacial layering was determined to be insignificant for larger particles. As the particles used are larger in size the interfacial effects of layering and convection are reduced and conduction models are sufficient to predict conductivity. Because nanoparticles are so small, interparticle surface forces become very important. The existence of the interparticle forces give rise to different energy modes for thermal transport. The Brownian Reynolds number is used to take into account the convective forces of the suspended nanoparticles. This is done by applying the Brownian Reynolds number to calculate the matrix thermal conductivity due to the convection caused by the movement of a single sphere.

Koo and Kleinstreuer [10] developed a model that uses the effects of the particle size, the particle volume fraction, and temperature dependence. Furthermore, the properties of base liquids and particle phase are considered to calculate the Brownian motion nanoparticles, as shown in the equation below,

$$
\left.k_{n f}=\left[\frac{k_{p}+2 k_{b f}-2 \phi\left(k_{b f}-k_{p}\right)}{k_{p}+2 k_{b f}+\phi\left(k_{b f}-k_{p}\right)}\right] k_{b f}+\left[5 \times 10^{4} \zeta \phi \rho_{b f} c_{b f} \sqrt{\frac{k_{B} T}{\rho_{p} d_{p}}} f(T, \phi, \text { etc. })\right]\right) k_{b f}
$$

Where

$$
f(T, \phi)=(-6.04 \phi+.4705) T+(1722.3 \phi-134.63)
$$

Where $\zeta$ is an empirical constant that is a function of the volume fraction of solute. Koo and Kleinstreur found the following $\zeta$ terms based on the experimental data they analysed

$\begin{array}{cccc}\begin{array}{c}\text { Type of } \\ \text { particle }\end{array} & \zeta & \text { Concentration } & \text { Temperature } \\ \mathrm{Al}_{2} \mathrm{O}_{3} & .0011(100 \phi)-.7272 & \phi \geq 1 \% & 300 K \leq \phi \leq 325 \mathrm{~K} \\ \mathrm{CuO} & .0017(100 \phi)-.0841 & \phi \geq 1 \% & 300 \mathrm{~K} \leq \phi \leq 325 \mathrm{~K}\end{array}$

They proposed that the enhanced thermal conductivity was due to micro-mixing that was induced due to Brownian motion which was effectively additive to the overall thermal conductivity of a static dilute suspension. Brownian motion is more predominant at higher temperatures due to the larger amounts of energy and vibration, which can be observed experimentally. To determine the thermal conductivity enhancement due to the Brownian motion, two nanoparticles were considered in the calculations. The two considered particles had time-averaged motion. They were then placed in two different temperature fields and the average distance for a particle to travel in one direction without changing paths was varied. To have a quantitative comparison of the micro-mixing and the 
induced heat transfer, the assumption of steady flow in the Stokes regime was made to be able to estimate the region of the affected fluid volume. However, the shape and size of the affected fluid volume depends on the particle shape. Hence, not only do the nanoparticles move due to Brownian motion, but the larger fluid body also moves, which leads to micro-mixing. Next, the interparticle potential was considered at high and low particle concentrations. Low concentrations show low dependency on thermal conductivity due to the lower amounts of particle interactions. Due to complexities of all considered effects the functions were determined through experimental analysis and best fit curves.

Vajiha and Das [14] renovated the empirical formulations for the Koo and Kleinstreuer [10] model. Vajiha and Das [14] found that the Koo and Kleinstreuer [10] matched their 133 experimental data points from three different types of nanofluids better than other existing Brownian models. However, Vajiha et al [14] were concerned with the range of validity for the Koo and Kleinstreuer [10] model. The Koo and Kleinstreuer [10] model was obtained from experiments on nanofluids within the $293 \mathrm{~K}<\mathrm{T}<325 \mathrm{~K}$ temperature range and $1 \%<\phi<4 \%$ concentration range. This led Vajiha and Das [10] to renovate the empirical correlations $\zeta$ and $f(T, \phi)$ from their larger set of experimental data. While using the same base thermal conductivity formula from equation 17 with the new empirical correlations shown as

$$
f\left(T, \phi_{p}\right)=(.028217 \phi+.003917)\left(\frac{T}{T_{0}}\right)+(-.030669 \phi-.00391123)
$$

and

$\begin{array}{cccc}\begin{array}{c}\text { Type of } \\ \text { particle }\end{array} & \beta & \text { Concentration } & \text { Temperature } \\ \mathrm{Al}_{2} \mathrm{O}_{3} & 8.4407(100 \phi)-1.07304 & 1 \% \leq \phi \leq 10 \% & 298 K \leq \phi \leq 363 \mathrm{~K} \\ \mathrm{ZnO} & 8.4407(100 \phi)-1.07304 & 1 \% \leq \phi \leq 7 \% & 298 K \leq \phi \leq 363 \mathrm{~K} \\ \mathrm{CuO} & 9.8810(100 \phi)-.9446 & 1 \% \leq \phi \leq 6 \% & 298 K \leq \phi \leq 363 \mathrm{~K}\end{array}$

Effectively Vajiha and Das [14] increased the range of validity for determining thermal conductivity of a nanofluid by expanding the range of temperature, concentration and nanoparticle type used.

Chon et al [15] proposed another empirical correlation focusing on the Brownian movement of particles suspended in a base fluid. They specifically focused on $\mathrm{Al}_{2} \mathrm{O}_{3}$ nanofluid data and developed their equation based on linear regression analysis as well as utilizing the Buckingham-Pi theorem. The correlation is known as

$$
k_{n f}=\left(1+64.7 \phi^{.746}\left(\frac{d_{b f}}{d_{n p}}\right)^{.369}\left(\frac{k_{n p}}{k_{b f}}\right)^{.746} \operatorname{Pr}^{.9955} \operatorname{Re}^{1.2321}\right) k_{b f}
$$

Where

And

$$
R e=\frac{\rho_{b f} k_{b} T}{3 \pi \mu_{b f}^{2} l_{b f}}
$$

$$
\operatorname{Pr}=\frac{c_{p b f} \mu_{b f}}{k_{b f}}
$$

Where $l_{b f}, \mu_{b f}$, and $c_{p b f}$ are the mean free path, dynamic viscosity and specific heat of the base fluid. A mean free path value of .17 $\mathrm{nm}$ is used throughout Chon et al's [15] paper throughout the entire range of temperature.

Patel et al [16] took a nonlinear regression over a large set of experimental data. Their analysis was based on using water, oil and ethylene glycol as base fluid. As well as using both $\mathrm{Al}_{2} \mathrm{O}_{3}$ and $\mathrm{CuO}$ nanoparticles. Patel et al [16] 
concluded that the reason for reduction in particle size enhancing thermal conductivity is closely due to high surface area and Brownian motion of particles [16]. As the size of nanoparticles is reduced the surface area per unit volume of nanoparticle increases. Heat transfer is dependent on surface area making the reduction in size enhance the heat transfer from nanoparticles to base fluid, thus increasing the thermal conductivity of the nanofluid as a whole. Their model is presented as

$$
k_{n f}=k_{b f}\left(1+0.135\left(\frac{k_{p}}{k_{b f}}\right)^{.273} \phi^{.467}\left(\frac{T}{20}\right)^{.547}\left(\frac{100}{d_{p}}\right)^{.234}\right)
$$

Where the validity range is with nanoparticles in size of 10-150nm, thermal conductivities of 20-400 $\left(\frac{\mathrm{W}}{\mathrm{mK}}\right)$, base fluids ranging from $0.1-0.7\left(\frac{W}{m K}\right)$, volume fractions ranging from $0.1 \%<\phi<3 \%$ and temperature ranging from 293 - 323 K. One important detail that in Patel et al's [16] equation the temperature and particle size are represented in Celsius and nanometres respectively.

Corcione [17] also considered Brownian movement as one of the main attributes to enhancing thermal conductivity using nanoparticles. Corcione [17] understood the difficulties in early models namely Maxwell [2] and Hamilton Crosser [3] models. Early models were unable to accurately predict thermal conductivity when tested under a range of temperature. This led to the investigation of more prominent variables effecting the thermal conductivity of nanofluids including the dimensionless Reynolds and Prandtl numbers of the base fluid at varying temperatures. Corcione [17] took data from over 13 different data sources including Chon et al [15], Eastman et al [20], Lee et al [11], and Murshed [33]. The data consisted of nanofluids ranging in material from $\mathrm{Al}_{2} \mathrm{O}_{3}, \mathrm{CuO}, \mathrm{TiO}_{2}$ as well as base fluids of both ethylene glycol and water and best fit the experimental data to come up with their model. Their model is given as

$$
k_{n f}=k_{b f}\left(1+4.4 \operatorname{Re}^{0.4} \operatorname{Pr}^{0.66}\left(\frac{T}{T_{f r}}\right)^{10}\left(\frac{k_{p}}{k_{b f}}\right)^{0.03} \phi^{0.66}\right.
$$

Where $T_{f r}$ is the freezing temperature of the base fluid. And Reynolds number is calculated as

$$
R e=\frac{2 \rho k_{b f} T}{\pi \mu_{b f}^{2} d_{p}}
$$

This model is restricted to the range of data that Corcione [17] used to propose his model. The validity range is Temperatures, $\mathrm{T}=294-324 \mathrm{~K}$, nanoparticle diameter $d_{p}=10-150 \mathrm{~nm}$, and volume fractions between $0.2 \%<\phi<9 \%$.

\section{THEORETICAL MODELLING OF THE VISCOSITY OF NANOFLUIDS}

When working with nanofluids, while a high thermal conductivity is desired in order to remove heat from the system another crucial property to take into consideration is the viscosity of the nanofluid. It is important due to the fact that pumping power can depend on the viscosity of the nanofluid being pumped. Throughout this section theoretical models for the approximation of viscosity will be discussed and individually explained. 
Some of the earliest research regarding fluid-particle mixtures was originally done by Einstein [21] in the early 1900's. Einsteins' [21] research focused on determining the effective viscosity of suspended spherical particles within a base fluid using hydrodynamic equations. Einstein's [21] generated a model as shown below in equation 26, where the viscosity of the mixture is dependent on the volume fraction concentration of particles within the base fluid.

$$
\mu_{n f}=1+2.5 \phi
$$

Where $\mu_{n f}$ is the effective viscosity of the mixture. This model does have its limitations, the Einstein [21] model tends to be valid for low particle concentrations of $0.02 \%$ by volume or less. Researchers such as Brinkman [22] and Lundgren [23] conducted further research in effort to extend the validity range of Einstein's [21] original equation. Brinkman [22] provided an improved revision of Einstein's' [21] original model shown below in equation 27. The Brinkman [22] model intended to extend the range of validity by increasing concentrations greater than $0.02 \%$.

$$
\mu_{n f}=\mu_{b f}\left(\frac{1}{(1-\phi)^{2.5}}\right)
$$

Where $\mu_{b f}$ is the viscosity of the base fluid. Brinkmann's [22] model can be used with moderate volume fraction concentrations. Lundgren [23] also proposed a new formula in the form of a Taylors Series, with viscosity being a function of the volume fraction concentration. In the Lundgren [23] model the terms above $\varphi^{2}$ are then negligible to the calculation of viscosity as shown below.

$$
\mu_{n f}=\mu_{b f}\left(1+2.5 \phi+\frac{25}{4} \phi^{2}+0 \phi^{3}\right)
$$

More recently researchers such as Maïga et al [24] have taken a more analytical approach using least square method against experimental data to determine appropriate formulas to predict viscosity of specific nanofluids. Maïga et al [24] considered the forced convection flow of $\mathrm{Al}_{2} \mathrm{O}_{3}$ nanoparticles suspended within both water and ethylene glycol base fluids. Maïga et al [24] formulated the model by curve fitting experimental data from Lee $e t$ al [11], Eastman [20] and Wang [34] and produced the formula shown in equation 29.

$$
\mu_{n f}=\mu_{b f}\left(1+7.3 \phi+123 \phi^{2}\right)
$$

Similarly, Nguyen et al [25] created best fit curves for calculating the effective viscosity of specific nanoparticles and sizes. Nguyen et al [25] conducted his own experiments using $\mathrm{Al}_{2} \mathrm{O}_{3}$ nanoparticles of $36 \mathrm{~nm}$ and $47 \mathrm{~nm}$ to determine the viscosity of the fluid with respect to concentration. Nguyen et al [25] then created correlations based off the gathered data for each respective size of alumina particles. He also conducted the same experiment with $\mathrm{CuO}$ particles of $29 \mathrm{~nm}$ in water and formulated an equation by best fit for this specific nanofluid. Nguyen et al [24] observed interesting trends in both the experimental data and the models themselves. The experimental data showed closely similar viscosities for $\mathrm{CuO}$ and $\mathrm{Al}_{2} \mathrm{O}_{3}$ from $0 \%$ concentration until about $4 \%$ where then the $\mathrm{CuO}-$ water nanofluid viscosity increases significantly and breaks away from the viscosity trend of the $\mathrm{Al}_{2} \mathrm{O}_{3}$-water fluid. Nguyen et al [24] believed this increased viscosity at higher concentrations of $\mathrm{CuO}$ could be due to the molecular structure of the mixture and the methods of dispersion within the base fluid. The correlations proposed by Nguyen et al [24] are shown for $47 \mathrm{~nm} \mathrm{Al}_{2} \mathrm{O}_{3}, 36 \mathrm{~nm} \mathrm{Al}_{2} \mathrm{O}_{3}$ and $29 \mathrm{~nm} \mathrm{CuO}$ respectively in equations 30-32.

$$
\begin{aligned}
\mu_{n f}= & \mu_{b f}\left(0.904 e^{0.148 \phi}\right) \\
& 47 \mathrm{~nm} \mathrm{Al}_{2} \mathrm{O}_{3}
\end{aligned}
$$




$$
\begin{gathered}
\mu_{n f}=1+0.025 \phi+0.015 \phi^{2} \\
36 \mathrm{~nm} \mathrm{Al}_{2} \mathrm{O}_{3} \\
\mu_{n f}=1.475-0.319 \phi+0.051 \phi^{2}+0.009 \phi^{3} \\
29 \mathrm{~nm} \mathrm{CuO}
\end{gathered}
$$

Rea et al [26] also conducted their own experiments, this time on nanofluids consisting of colloidal $\mathrm{Al}_{2} \mathrm{O}_{3}$ and $\mathrm{ZnO}$ nanoparticles of $50 \mathrm{~nm}$ in water. Rea et al [26] used the transient hot wire method and varied the temperature of their channel in order to collect thermo physical properties of the fluids. Therefore, making their model as a function of both concentration and varying temperature. They then logged the data and created a best fit curve for each specific mixture of fluids. Their correlations shown below in equations 33 and 34 are for $\mathrm{Al}_{2} \mathrm{O}_{3}$ and $\mathrm{ZnO}$ within water respectively.

$$
\begin{gathered}
\mu_{n f}=\mu_{b f} e^{(4.91 \phi / 0.2092-\phi)} \\
\mu_{n f}=\mu_{b f}\left(1+46.801 \phi+550.82 \phi^{2}\right)
\end{gathered}
$$

Where the viscosity of the base fluid varies with temperature. The $\mathrm{Al}_{2} \mathrm{O}_{3}$ formula is valid from $0 \%$ to $6.0 \%$ concentrations while the $\mathrm{ZnO}$ model is valid from $0 \%$ to $3 \%$.

Khanafer and Vafai [27] were able to develop three equations, calculating viscosity as functions of both temperature and volume fraction. Khanafer and Vafai [27] believed previous models were lacking temperature as a parameter to be considered when calculating the effective viscosity of a nanofluid. The models were developed by using a least-square regression analysis, based off experimental data from Nguyen et al [25], Pak and Cho [30], Anoop et al [32], and Putra et al [31]. The data gathered from these various sources were of nanofluids consisting of $36 \mathrm{~nm}$ and $47 \mathrm{~nm} \mathrm{Al} \mathrm{Al}_{3}$ as well as $\mathrm{TiO}_{2}$ and $\mathrm{CuO}$ of various sizes. All with water as base fluid and tested under varying temperature. A curve fitting expression to the data of $\mathrm{Al}_{2} \mathrm{O}_{3}$-Water nanofluids $[25,30,31,32]$ is proposed by Khanafer and Vafai [27]. Where the viscosity is dependent on the volume fraction, temperature and the size of the nanoparticle as seen in equation 35 .

$$
\begin{aligned}
\mu_{n f}=-0.4491 & +\frac{28.4312}{T}+0.574 \phi-0.1634 \phi^{2}+\frac{23.053 \phi^{2}}{T^{2}}+0.0132 \phi^{3}-\frac{2354.735 \phi}{T^{3}} \\
+ & \frac{23.498 \phi^{2}}{d_{p}^{2}}-\frac{3.0185 \phi^{3}}{d_{p}^{2}}
\end{aligned}
$$

This equation is valid for a volume fraction range from $1 \%-9 \%$ and a temperature range from $20_{\circ} \mathrm{C}-70_{\mathrm{o}} \mathrm{C}$.

Abu-Nada [28] much like Khanafer and Vafai [26] was concerned with the lack of consideration of temperature in calculating the dynamic viscosity of nanofluids. Abu-Nada [28] looked closely at data and correlations proposed by Nguyen et al [25] and developed new curve fitting models in order to incorporate the effects of changing temperature on viscosity. Therefore, generating a new model where viscosity is dependent on both the volume fraction concentration and temperature based off the collected data from Nguyen et al [25]. Abu-Nada [28] proposed the following model through regression analysis with an $\mathrm{R}_{2}$ value of $99.8 \%$ and maximum error of 5\% with respect to the Nguyen et al [25] experimental data. The formula proposed is shown below in equation 36. 


$$
\begin{aligned}
\mu_{n f}=-0.155 & -\frac{19.582}{T}+0.794 \phi \frac{+2094.47}{T^{2}}-0.192 \phi^{2}-8.11 \frac{\phi}{T}-\frac{27463.863}{T^{3}}+0.0127 \phi^{3} \\
& +1.6044 \frac{\phi^{2}}{T}+2.175 \frac{\phi}{T^{2}}
\end{aligned}
$$

These equations will be compared to experimental data from various sources in order to observe general trends of viscosity with respect to temperature, size, and concentration. As well as examine the level of accuracy of the models to the experimental data.

\section{EXAMINATION OF THEORETICAL MODELS WITH EXISTING DATA}

This section will examine the accuracy of theoretical models approximating thermal conductivity of nanofluids as well as showing some general trends of both viscosity and thermal conductivity due to size, concentration and temperature. The accuracy is examined by comparing proposed theoretical models with experimental data and calculating an average percent error of each model. It is crucial that models are compared to third party experimental data in order to remove potential bias in research papers. By comparing different sets of experimental data against the selected theoretical models, it is possible to quantitatively select the best theoretical model for the specific set of experimental data. Theoretical models are tailored to specific conditions and properties of the nanofluid composition, deviating from a model's specific characteristics will significantly decrease accuracy of the approximations. This section will explore determining some of the most accurate models in calculating thermal conductivity of nanofluids in certain situations.

\subsection{Effective Medium Theory Models Comparison}

Table 1 shows the average percent error of EMT based methods with varying experimental data. All the presented models are rooted from the standard effective medium theory initially proposed by Maxwell [2]. The models all assume the static and homogenous dispersion of the nanoparticle within the nanofluid. These models are compared side by side in order to determine the most accurate model for certain nanofluids.

Table 1: Average percent error of EMT based methods with varying experimental data.

\begin{tabular}{|c|c|c|c|c|c|}
\hline Reference & \multicolumn{5}{|c|}{$\begin{array}{c}\text { Average Percent Error [\%] } \\
\text { EMT Methods }\end{array}$} \\
\hline $\begin{array}{c}\text { Sundar } \\
{[5]}\end{array}$ & $\begin{array}{c}\text { Maxwell } \\
{[2]}\end{array}$ & $\begin{array}{c}\text { Hamilton } \\
\text { Crosser } \\
\text { Spheres } \\
{[5]}\end{array}$ & $\begin{array}{c}\text { Hamilton } \\
\text { Crosser } \\
\text { Cylinders } \\
{[5]}\end{array}$ & $\begin{array}{c}\text { Wasp } \\
\text { et al. } \\
{[41]}\end{array}$ \\
\hline $\begin{array}{c}\text { Water- } \\
\mathrm{Al}_{2} \mathrm{O}_{3},[11]\end{array}$ & 5.74 & 0.793 & 0.793 & --- & --- \\
\hline $\begin{array}{c}\text { Water-CuO } \\
{[11]}\end{array}$ & 5.54 & 1.39 & 1.39 & --- & --- \\
\hline $\begin{array}{c}\mathrm{EG}-\mathrm{Al}_{2} \mathrm{O} 3 \\
{[11]}\end{array}$ & 6.73 & .90 & .90 & 1.03 & .91 \\
\hline $\begin{array}{c}\mathrm{EG}-\mathrm{CuO} \\
{[11]}\end{array}$ & 6.10 & 3.17 & 3.17 & 3.29 & 3.16 \\
\hline $\begin{array}{c}\text { Water-ZnO } \\
{[12]}\end{array}$ & --- & 6.46 & 6.5 & 5.54 & 6.46 \\
\hline $\begin{array}{c}\text { Water-TiO } 2 \\
{[12]}\end{array}$ & --- & 8.20 & 8.25 & 7.27 & 8.21 \\
\hline $\begin{array}{c}\text { Water- } \\
\mathrm{Al} 2 \mathrm{O}_{3}[12]\end{array}$ & --- & 10.58 & 10.6 & 9.18 & 10.59 \\
\hline
\end{tabular}




\begin{tabular}{|c|c|c|c|c|c|}
\hline $\begin{array}{c}\text { Water- } \\
\mathrm{Al}_{2} \mathrm{O}_{3} \\
{[11]}\end{array}$ & --- & 1.45 & 1.68 & 8.77 & 1.46 \\
\hline $\begin{array}{c}\text { Water- } \\
\mathrm{Fe}_{3} \mathrm{O}_{4} .2 \% \\
\text { Vol } \\
\text { Fraction [5] }\end{array}$ & .553 & 9.42 & 9.43 & 9.38 & 9.42 \\
\hline $\begin{array}{c}\text { Water- } \\
\mathrm{Al}_{2} \mathrm{O}_{3}[7]\end{array}$ & --- & --- & 7.47 & 2.96 & -- \\
\hline
\end{tabular}

Fig. 5 shows experimental data obtained for $\mathrm{CuO}$ nanoparticles in ethylene glycol base fluid from Lee et al [11]. For this set of data, the most accurate models have been determined to be the Maxwell [2], Hamilton Crosser [3] under the spherical nanoparticle assumption, and the Wasp et al [4]. The average percent error for these three equations was $3.17 \%$ due to the close similarity between these models and the relatively low concentration of particles the average percent error was the same. The Wasp et al [4] model is known to produce results very similar to Hamilton Crosser [3] with spherical nanoparticles. The Sundar et al [5] model was relatively inaccurate at an average percent error of $6.1 \%$; the Sundar [4] model was used in order to show the inaccuracies incurred from using a model outside of its specified bounds.

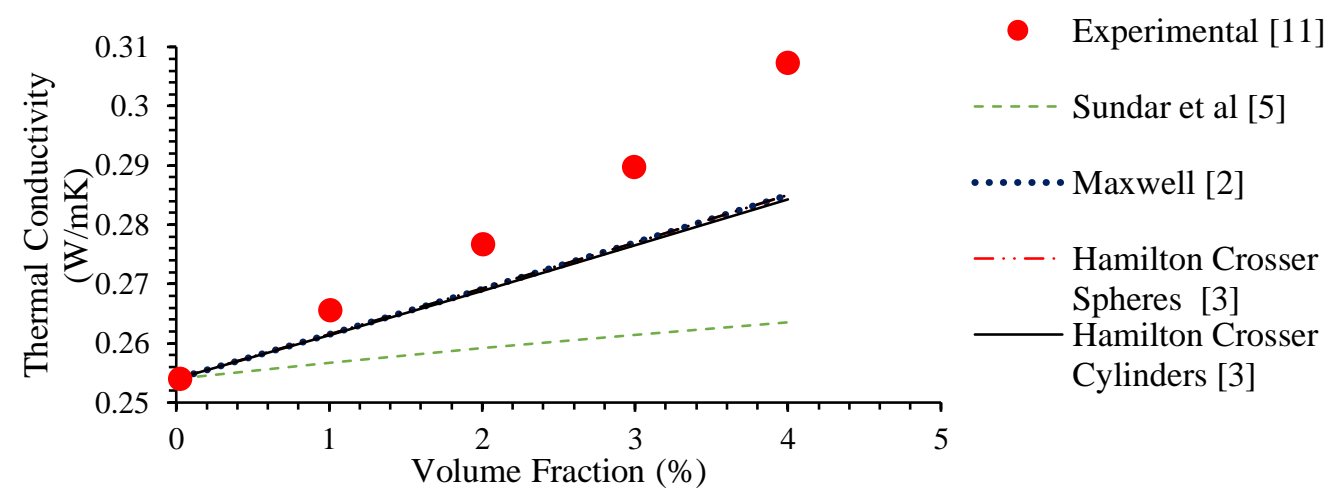

Fig. 5 Thermal Conductivity vs Volume Fraction of $\mathrm{CuO}$ nanoparticles in ethylene glycol base fluid with EMT models.

The thermal conductivity as a function of temperature is shown in Fig. 6. The general trend is as temperature increases the thermal conductivity consequently increases. Sundar et al s varied the temperature at which the nanofluid is tested ranging from 20 to 60 degrees Celsius as well as increased the volume fraction from $0.2 \%$ to $2.0 \%$. In both cases as the temperature and volume fraction increase the enhancement in thermal conductivity is greater. Sundar is able to best approximate the thermal conductivity under these varying temperature conditions in this case as shown in Fig. 6 while other models including Maxwell [2], Wasp et al [4] and Hamilton Crosser [11] significantly underestimate the thermal conductivity under the variance of temperature. The Sundar [5] model had an average percent error of only $0.55 \%$ making it the most accurate model for the prediction of thermal conductivity with varying temperature. It is good to note that the Sundar [5] model is bounded by the range of temperatures and concentration of nanoparticles that were used in the experiment. Where $0<\phi<2.0 \%, 20^{\circ} \mathrm{C}<\mathrm{T}$ $<60^{\circ} \mathrm{C}$. 


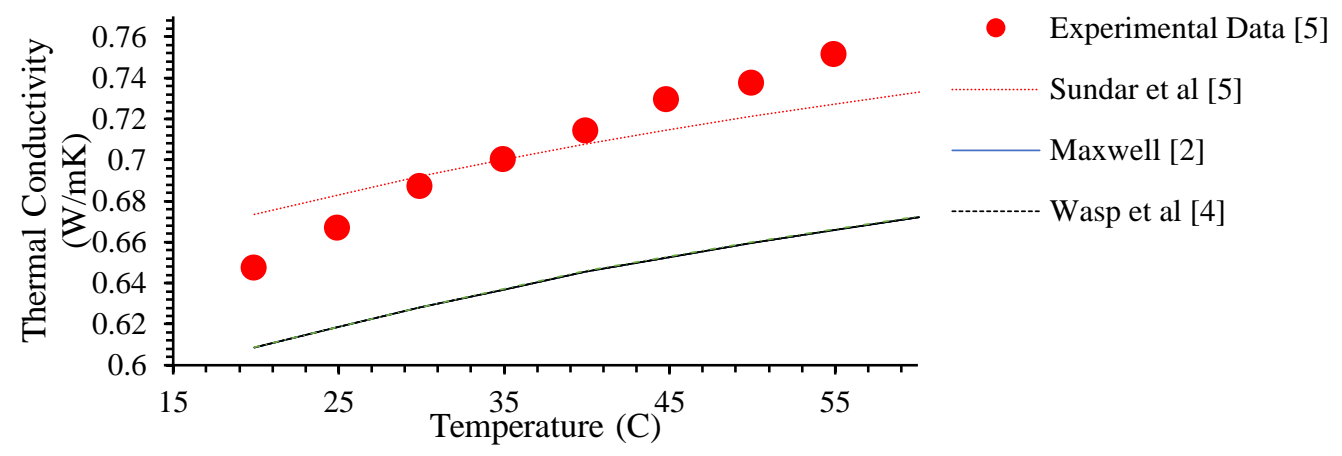

Fig. 6 Thermal Conductivity vs Temperature of $\mathrm{CuO}$ nanoparticles in ethylene glycol base fluid with EMT models.

\subsection{Nanolayer Theory Models Comparison}

Table 2 shows the average percent error of Nanolayer based theoretical models with varying experimental data from several sources. The nanolayer was built off the original effective medium theory model and added the characterization of the layering effect on the nanoparticle due to the base fluid-nanoparticle interactions. The nanolayer method insists that the driving factor for thermal conductivity enhancement is due to the layer formed around the nanoparticle where the thermal conductivity in this layer is in between that of the nanoparticle and base fluid.

Table 2: Average percent error of Nanolayer based methods with varying experimental data.

\begin{tabular}{|c|c|c|c|c|c|}
\hline \multicolumn{6}{|c|}{$\begin{array}{l}\text { Average Percent Error [\%] } \\
\text { Nanolayer Methods }\end{array}$} \\
\hline References & $\begin{array}{l}\text { Leong [7] } \\
k_{n l} \\
=2 k_{b f}\end{array}$ & $\begin{array}{l}\text { Leong [7] } \\
k_{n l} \\
=3 k_{b f}\end{array}$ & $\begin{array}{c}\text { Yu and } \\
\text { Choi [8] }\end{array}$ & Xie [6] & Tinga et al [13] \\
\hline $\begin{array}{l}\text { Water- 80nm } \\
\mathrm{Al}_{2} \mathrm{O}_{3}[5]\end{array}$ & 3.30 & 7.78 & 5.99 & 7.59 & 12.98 \\
\hline $\begin{array}{c}\text { Water- } \\
\text { 150nm } \\
\mathrm{Al}_{2} \mathrm{O}_{3}[5]\end{array}$ & 1.33 & .716 & 1.99 & 2.02 & 2.75 \\
\hline $\begin{array}{c}\text { EG- } 150 \mathrm{~nm} \\
\mathrm{Al}_{2} \mathrm{O}_{3} \\
{[6]}\end{array}$ & 1.04 & 9.04 & 6.00 & 7.63 & 15.1 \\
\hline $\begin{array}{c}\text { EG- } \\
150 \mathrm{~nm} \\
\mathrm{Al}_{2} \mathrm{O}_{3}[11]\end{array}$ & 7.98 & 17.69 & 1.24 & .84 & 7.56 \\
\hline $\begin{array}{c}\text { Water- } \mathrm{CuO} \\
{[31]}\end{array}$ & 12.2 & 6.64 & 18.2 & 18.4 & 23.1 \\
\hline $\begin{array}{c}\text { EG- } \\
\mathrm{CuO}[11]\end{array}$ & 2.34 & 10.3 & 3.16 & 4.38 & 10.2 \\
\hline
\end{tabular}




\begin{tabular}{|c|c|c|c|c|c|}
\hline $\begin{array}{c}\text { Water- } \\
\mathrm{Al}_{2} \mathrm{O}_{3},[11]\end{array}$ & --- & ---- & 7.19 & 0.708 & -- \\
\hline $\begin{array}{c}\text { Water-CuO } \\
{[11]}\end{array}$ & --- & --- & 3.37 & 1.64 & --- \\
\hline
\end{tabular}

Experimental data is taken with the proper constants in order to compare several different Nanolayer theoretical models. The nanolayer theory further characterizes the heat transfer throughout the nanofluid in efforts to more accurately predict the effective thermal conductivity of the working fluid. Several sets of data were taken from Leong et al [5] experimental findings with varying nanoparticle sizes, base fluids, and nanoparticles. In the first case in the Table 2 Leong et al [5] experimentally determined the thermal conductivity of $80 \mathrm{~nm} \mathrm{Al}_{2} \mathrm{O}_{3}$ via the transient hot-wire method at room temperature and atmospheric pressure. The nanolayer thickness is assumed to be $1 \mathrm{~nm}$, the particle radius being $16.5 \mathrm{~nm}$ and the nanolayer thermal conductivity, $k_{n l}$, is modelled at $2 k_{b f}$ and $3 k_{b f}$ for the Leong et al [5] model. This experimental data is compared with 5 different nanolayer methods in Fig. 12. The average percent error is taken and the Leong et al [5] model under the $k_{n l}=2 k_{b f}$ assumption is in the best agreement with the experimental data presented at only $3.3 \%$ average error over 5 data points. The Yu and Choi model is also similarly accurate assuming $k_{p e}=k_{n p}$ with an average percent error of 5.99\%, the Xie [6] and Tinga et al [13] model fall under the least accurate models for this set of experimental data consistently under estimating the thermal conductivity of nanofluid at $7.6 \%$ and $12.98 \%$ average error respectively.

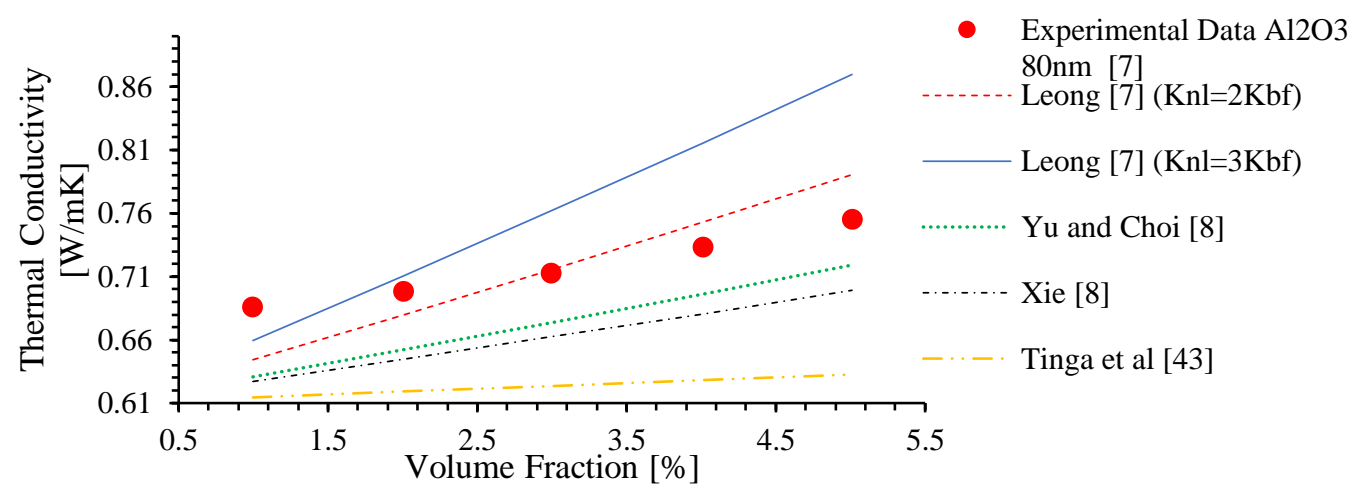

Fig. 7 Thermal Conductivity vs. Volume Fraction using $80 \mathrm{~nm} \mathrm{Al}_{2} \mathrm{O}_{3}$ in water with nanolayer models.

Similarly, Fig. 5 shows the presented nanolayer models compared to experimental data using $28.6 \mathrm{~nm} \mathrm{CuO} \mathrm{in} \mathrm{an}$ ethylene glycol base fluid. The same assumptions made in Fig. 7 are applied to the nanolayer methods. For this set of experimental data the most accurate model was determined to be the Leong et al [5] model with an average percent error $2.34 \%$ followed by the Yu and Choi [6] at 3.16\%. Tinga et al [13] and the Leong et al [5] $k_{n l}=$ $3 k_{b f}$ resulted in average percent errors of over $10 \%$. 


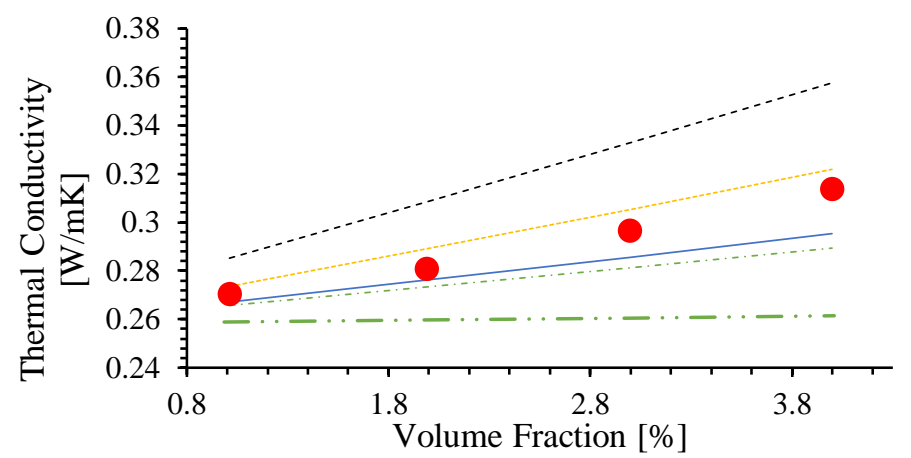

- Experimental $\mathrm{CuO}$ [11]

Xie [6]

Leong [7] (Knl=2Kbf)

Yu and Choi [8]

— - - Tinga et al [13]

Leong [7] (Knl=3Kbf)

Fig. 8 Thermal Conductivity vs. Volume Fraction using $28.6 \mathrm{~nm} \mathrm{CuO} \mathrm{nanoparticles} \mathrm{in} \mathrm{Ethylene} \mathrm{Glycol} \mathrm{with}$ nanolayer models.

\subsection{Brownian Models Comparison}

Table 3 shows the average error percent of Brownian based models compared to experimental data. Brownian models take into consideration the random motion of particles suspended throughout a liquid as well as the localized convection due to this movement. The current models being evaluated are the Prasher et al [9], Koo and Kleinstreuer [10], Vajiha and Das [14] and the Chon et al [15] models.

Table 3 Average percent error of Brownian based methods with varying experimental data.

\begin{tabular}{|c|c|c|c|c|c|}
\hline \multicolumn{6}{|c|}{$\begin{array}{c}\text { Average Absolute Error [\%] } \\
\text { Brownian Models }\end{array}$} \\
\hline References & $\begin{array}{c}\text { Koo and } \\
\text { Kleinstreur [10] }\end{array}$ & $\begin{array}{c}\text { Vajiha and } \\
\text { Das [14] }\end{array}$ & $\begin{array}{c}\text { Chon et al } \\
\text { [15] }\end{array}$ & $\begin{array}{c}\text { Corcione } \\
{[17]}\end{array}$ & $\begin{array}{l}\text { Patel et al } \\
\quad[16]\end{array}$ \\
\hline $\begin{array}{c}\text { Water-28.6nm } \\
\quad \mathrm{CuO}[9] \\
\phi=1 \%\end{array}$ & 1.64 & 5.39 & --- & --- & --- \\
\hline $\begin{array}{l}\text { Water-28.6nm } \\
\quad \mathrm{CuO}[9] \\
\phi=4 \%\end{array}$ & 2.44 & 2.81 & --- & --- & --- \\
\hline $\begin{array}{c}\text { Water-38.4nm } \\
\mathrm{Al}_{2} \mathrm{O}_{3}[18] \\
\phi=1 \%\end{array}$ & 21.73 & 3.66 & 1.84 & .467 & 1.51 \\
\hline $\begin{array}{c}\text { Water-38.4nm } \\
\mathrm{Al}_{2} \mathrm{O}_{3}[18] \\
\phi=4 \%\end{array}$ & 40.19 & 2.97 & 1.74 & .568 & 2.39 \\
\hline $\begin{array}{c}\text { Water-29.8nm } \\
\text { CuO [14] } \\
\phi=4 \%\end{array}$ & 6.56 & 1.37 & --- & 15.4 & --- \\
\hline $\begin{array}{c}\text { Water-47nm } \\
\mathrm{Al}_{2} \mathrm{O}_{3}[15] \\
\phi=4 \%\end{array}$ & 51.8 & 3.26 & 1.07 & 4.98 & 2.93 \\
\hline $\begin{array}{c}\text { Water-11nm } \\
\mathrm{Al}_{2} \mathrm{O}_{3}[15] \\
\phi=1 \%\end{array}$ & --- & 8.43 & 2.36 & 5.57 & 0.57 \\
\hline $\begin{array}{l}\text { Water-47nm } \\
\mathrm{Al}_{2} \mathrm{O}_{3}[15]\end{array}$ & 29.84 & 1.77 & 0.39 & 3.03 & 1.76 \\
\hline
\end{tabular}




\begin{tabular}{|c|c|c|c|c|c|}
\hline$\phi=1 \%$ & & & & & \\
\hline $\begin{array}{c}\text { Water-150nm } \\
\mathrm{Al}_{2} \mathrm{O}_{3}[15] \\
\phi=1 \%\end{array}$ & 18.27 & 1.60 & 0.84 & 3.53 & 2.53 \\
\hline $\begin{array}{c}\text { Water-23.6nm } \\
\mathrm{CuO}[18] \\
\mathrm{T}=293 \mathrm{~K}\end{array}$ & 2.51 & 2.51 & --- & 2.04 & 0.68 \\
\hline $\begin{array}{c}\text { Water-38.4nm } \\
\mathrm{Al} 2 \mathrm{O} 3[11] \\
\mathrm{T}=293 \mathrm{~K}\end{array}$ & 0.37 & 0.92 & 2.43 & 2.06 & 1.99 \\
\hline $\begin{array}{c}\text { Water-33nm } \\
\mathrm{Al} 2 \mathrm{O} 3[20] \\
\mathrm{T}=293 \mathrm{~K}\end{array}$ & 8.15 & 7.30 & 14.4 & 11.8 & 6.79 \\
\hline $\begin{array}{c}\text { Water-23.6nm } \\
\mathrm{CuO}[18] \\
\phi=1 \%\end{array}$ & 1.57 & 6.33 & --- & 9.12 & 9.50 \\
\hline $\begin{array}{c}\text { Water-23.6nm } \\
\mathrm{CuO}[18] \\
\phi=4 \%\end{array}$ & 1.03 & 2.69 & --- & 3.20 & 7.81 \\
\hline
\end{tabular}

Fig. 9 shows several Brownian models being compared to experimental data gathered using $28.6 \mathrm{~nm} \mathrm{CuO}$ nanoparticles in a base fluid of water with volume fractions of $1 \%$ and $4 \%$ by Prasher et al [9]. Using the Prasher model [9] to predict the thermal conductivity requires the definition of several constants. $\mathrm{m}, \mathrm{Rb}$ and $\mathrm{A}$ all must be defined in order to calculate the Brownian thermal conductivity. $\mathrm{m}$ and $\mathrm{A}$ are the best fit constant and the multiplier constant respectively. $\mathrm{m}$ is determined to be 2.35 for the $\mathrm{CuO}$ volume fraction $\phi=4 \%$ and $\mathrm{m}=2.05$ for $\phi=1 \%$ through best fit analysis done by Prasher et al [9]. A is a multiplier of 40,000 in this case. Rb, the thermal boundary resistance is assumed to be $.77 * 10^{-8}\left[\frac{\mathrm{Km}^{2}}{\mathrm{~W}}\right]$. The kinematic viscosity, thermal conductivity of the base fluid, and Prandtl number of the base fluid are all functions of temperature. Therefore, their values also vary with changing temperature throughout the calculations of the nanofluid thermal conductivity. In the case of Koo and Kleinstreur [10] calculations, $\beta$ and $f\left(T, \phi_{p}\right)$ are defined by Koo and Kleinstreuer as their empirical functions of best fit with the data they analysed [10]. Vajiha and Das [14] renovated Koo and Kleinstreur's [10] experimental functions of $\beta$ and $f\left(T, \phi_{p}\right)$ through the analysis of a wider range of experimental data increasing the bounds of validity for the Vajiha and Das [14] model for Brownian thermal conductivity.

The most accurate model compared to the experimental data in Fig. 9 was determined to be the Prasher et al [9] model with an average error percent of $1.61 \%$. Koo and Kleinstreur [10] as well as Vajiha et al [14] models incurred similar average error percent's of $2.44 \%$ and $2.81 \%$ respectively. When another set of experimental data [9] was used with the same nanoparticle but with a lower concentration (1\%), Koo and Kleinstreur [10] more accurately predicts the thermal conductivity with an average error percent of only $1.64 \%$ while Prasher [9] and Vajiha [14] models incur $2.3 \%$ and $5.48 \%$ errors.

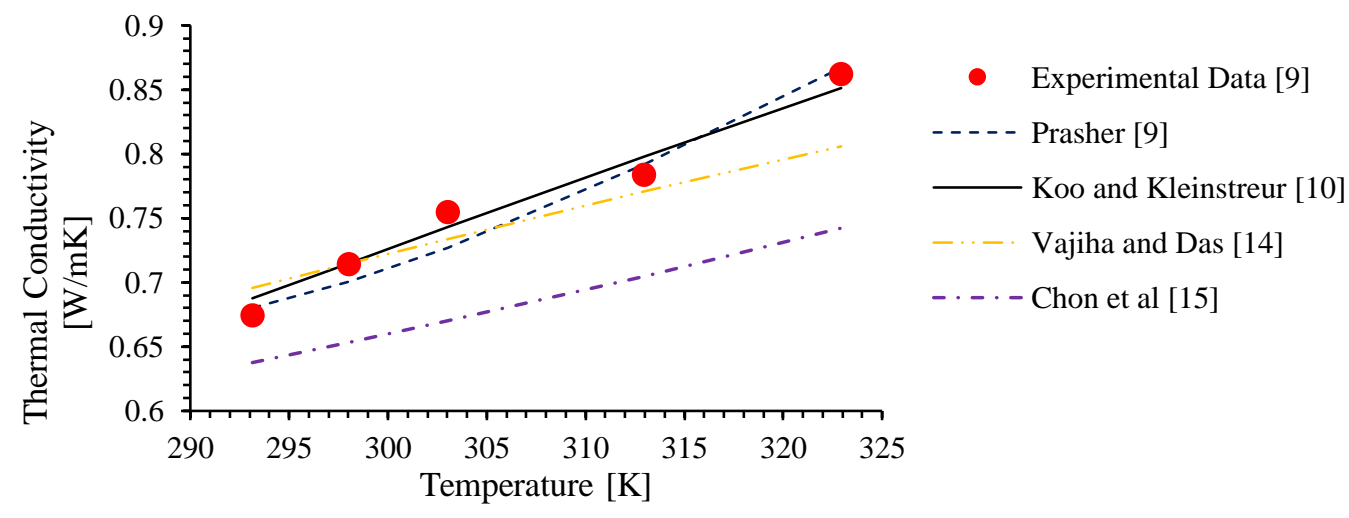


Fig. 9 Thermal Conductivity vs. Temperature using 28.6nm CuO nanoparticles with fixed volume fraction of $4 \%$.

Fig. 10 utilizes data from Das et al [14] where $38.4 \mathrm{~nm} \mathrm{Al}_{2} \mathrm{O}_{3}$ particles are used in a water based nanofluid. The concentration of particles is fixed at $4 \%$ while the temperature is varied from $273 \mathrm{~K}-323 \mathrm{~K}$. An upwards trend of thermal conductivity with respect to temperature is apparent in the experimental data. The Brownian models shown are in good agreement in terms of predicting the thermal conductivity of the nanofluid. The Corcione [17] model most accurately estimates the thermal conductivity with an average absolute error of only $0.56 \%$. The Chon et al [15], Patel et al [16] and, Vajiha and Das [14] models also closely predict the experimental data with average errors of only $1.74 \%, 2.39 \%$ and $2.97 \%$ respectively. Table 3 as well as the Figures 9 and 10 show the Brownian models more accurately predict the thermal conductivity of various nanofluids from several third-party experimentations.

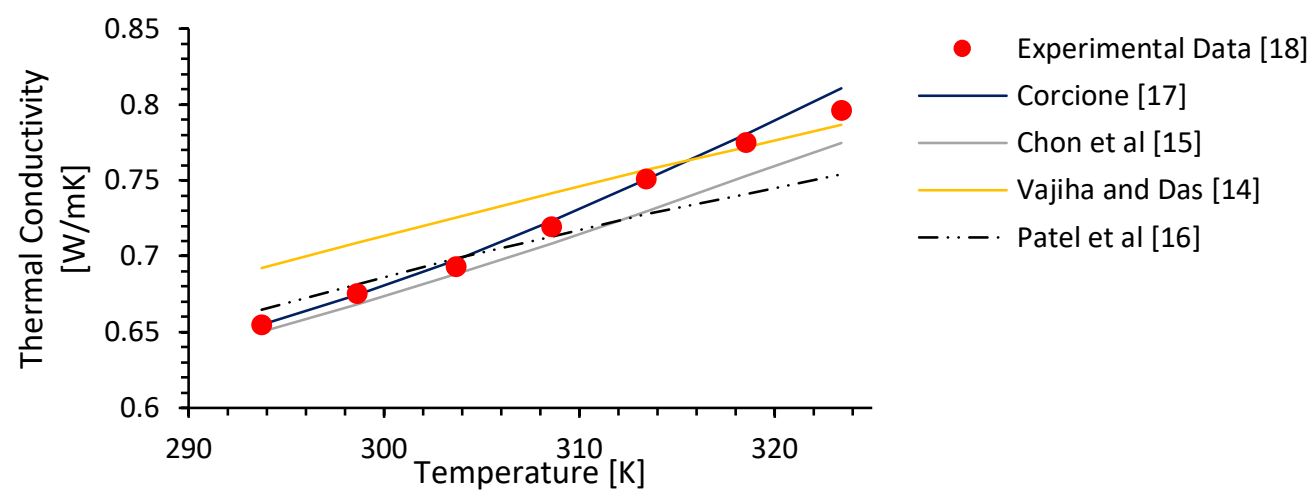

Fig. 10 Thermal Conductivity vs. Temperature using 38.4nm $\mathrm{Al}_{2} \mathrm{O}_{3}$ nanoparticles with fixed volume fraction of $4 \%$.

More experimental data is gathered from Das [18] of both $38.4 \mathrm{~nm} \mathrm{Al}_{2} \mathrm{O}_{3}-\mathrm{W}$ ater and 23.6nm CuO-Water nanofluids at concentrations of $1 \%$ and $4 \%$ by volume in order to see the effect on thermal conductivity of different concentrations. An observation can be made from both Fig. 11 and Fig. 12 on the impact of concentration on the thermal conductivity. As the concentration of the nanoparticle increases the thermal conductivity consequently increases regardless of material. In both cases of the $\mathrm{Al}_{2} \mathrm{O}_{3}$ and $\mathrm{CuO}$ the $4 \%$ concentration by volume has an increased thermal conductivity relative to the $1 \%$. This can be due to the increased presence of the higher thermally conductivity particles throughout the base fluid, increasing the thermal conductivity of the nanofluid. Corcione [17] and Chon et al [15] models are used to approximate the gathered experimental data of the $\mathrm{Al}_{2} \mathrm{O}_{3}-\mathrm{Water}$ nanofluid from Das [18]. The models are in close agreement with the data with absolute average errors of only $0.47 \%$ and $1.74 \%$ respectively. In the case of the $\mathrm{CuO}$-Water nanofluids the Koo and Kleinstreur [10] and Vajiha and Das [14] models are used to predict the experimental data, they do so closely with absolute average errors of $1.56 \%$ and $2.69 \%$ respectively. 


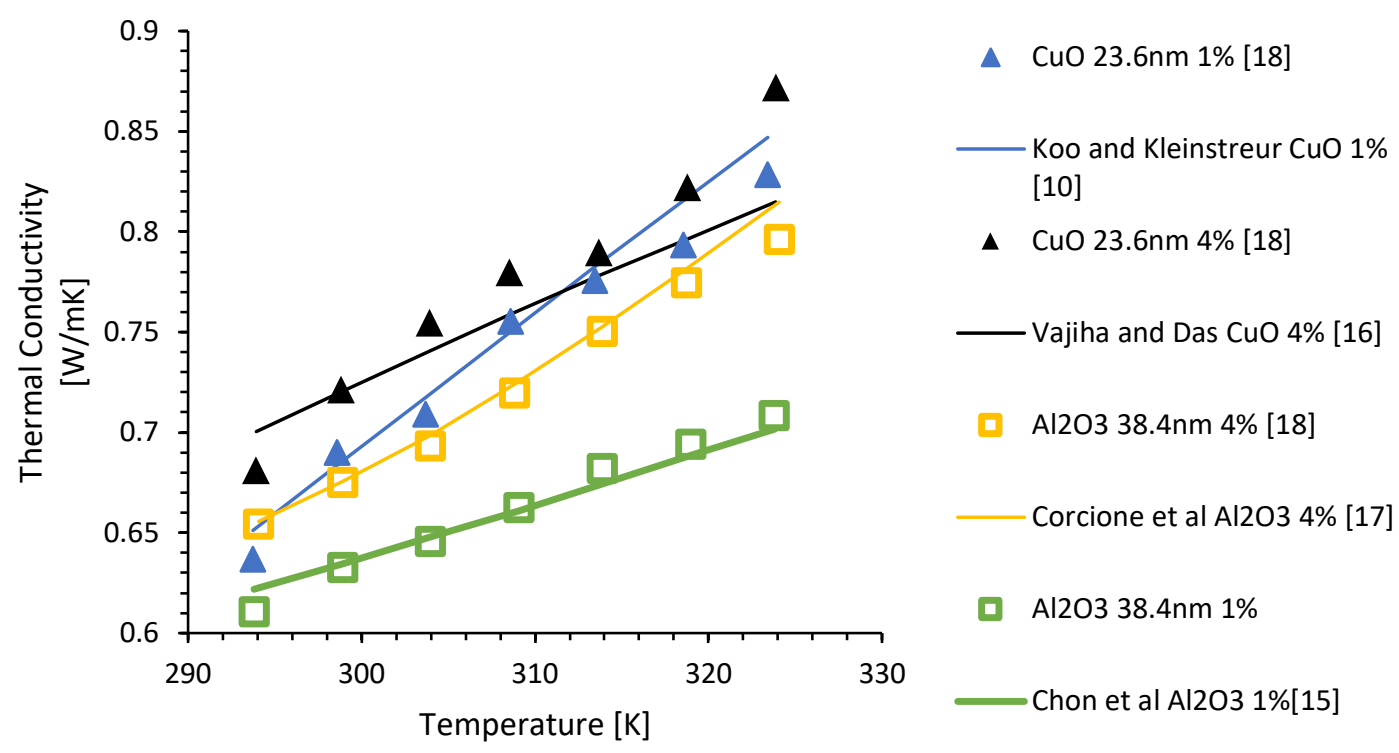

Fig. 11 Thermal Conductivity vs. Temperature using $38.4 \mathrm{~nm} \mathrm{Al}_{2} \mathrm{O}_{3}$ and $23.6 \mathrm{~nm} \mathrm{CuO}$ at concentrations of $1 \%$ and $4 \%$.

Shown in Fig. 12 is experimental data taken from Chon et al [15] of $\mathrm{Al}_{2} \mathrm{O}_{3}$ - Water nanofluids, varying in particle sizes and concentrations. The sizes of particles are $11 \mathrm{~nm}, 47 \mathrm{~nm}$ and $150 \mathrm{~nm}$. The thermal conduvtivities of the $11 \mathrm{~nm}$ and $150 \mathrm{~nm} \mathrm{Al}_{2} \mathrm{O}_{3}$ nanofluids were measured at $1 \%$ volume fraction. While the $47 \mathrm{~nm} \mathrm{Al}_{2} \mathrm{O}_{3}$ nanofluid was measured at both $1 \%$ and $4 \%$ particle volume fraction. Brownians models including Patel et al [16], Chon et al [15], Vajiha and Das [14] as well as Corcione [17] all estimate the separate sets data with less than 5\% absolute average error. At $1 \%$ concentration the smallest particle $(11 \mathrm{~nm})$ had the largest thermal conductivity enhancement of the three measured nanofluids followed by the $47 \mathrm{~nm}$ particles and finally the $150 \mathrm{~nm}$. Showing a general trend of greater enhancement due to reduction in particle size. The $47 \mathrm{~nm}$ particle with $4 \%$ concentration had the highest thermal conductivity produced of any nanofluid. The increased concentration significantly impacts the thermal conductivity of the nanofluid.

$\mathrm{Al}_{2} \mathrm{O}_{3}$ - water nanofluids are some of the most commonly used nanofluids in experimentation today. The equations presented in Fig. 12 all closely predict the experimental values by taking into consideration brownian motion of particles in the basefluid. In the case of the $11 \mathrm{~nm}$ particles with $1 \%$ volume fraction concentration within the basefluid, the Patel et al [16] equation predicts the experimental values with only $0.57 \%$ absolute average error. Similarly with the $47 \mathrm{~nm}$ particles with $4 \%$ concentration the Chon et al [15] model predicts the thermal conductivity of the fluid with an absolute error of $1.06 \%$. The remaining errors produced by the models are showin in Table 3. 
TFEC-2020-32505

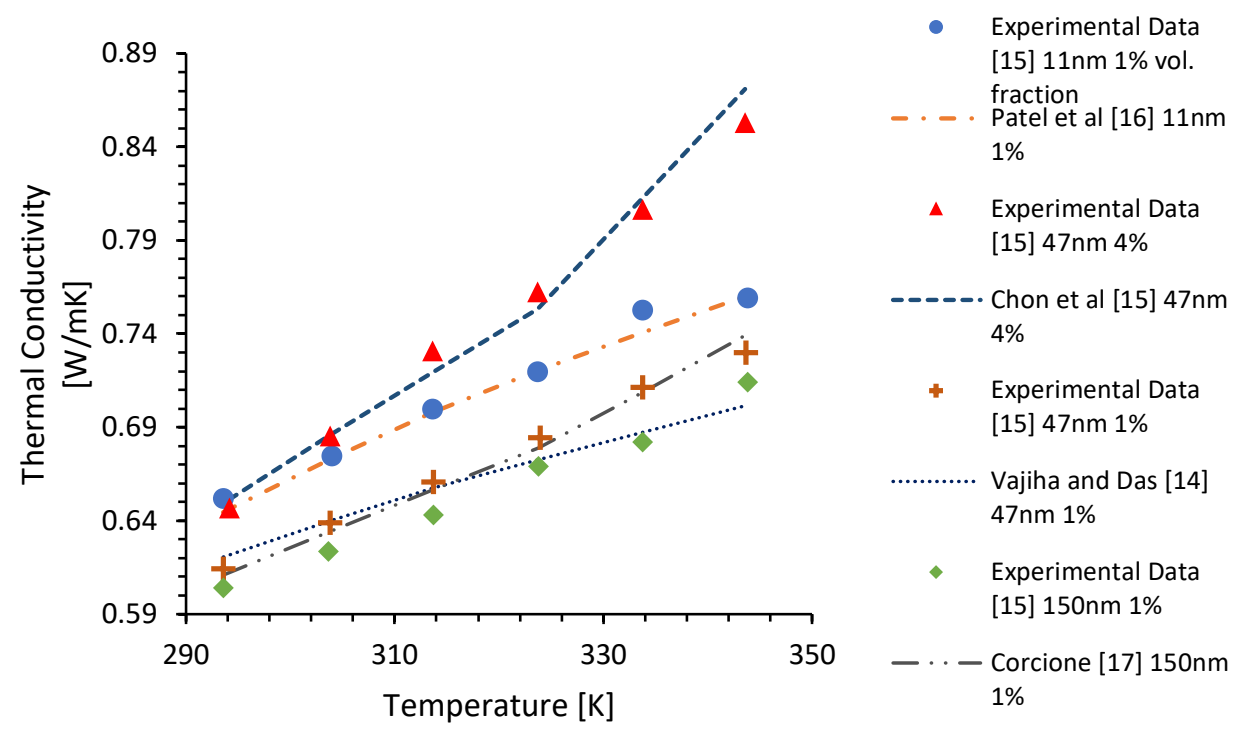

Fig. 12 Comparison of different Experimental Data for various sizes of $\mathrm{Al}_{2} \mathrm{O}_{3}$-water nanofluids [15] to Brownian models.

\subsection{Effects of Nanoparticles on Viscosity and Thermal Conductivity of Nanofluids}

The impact of concentration by volume fraction on thermal conductivity and viscosity of nanofluids can be observed in Fig. 13. The figure shows data acquired from Das [18], Lee et al [11] and Pastorgoriza-Gallego [29] of $38.4 \mathrm{~nm} \mathrm{Al}_{2} \mathrm{O}_{3}, 23.6 \mathrm{~nm} \mathrm{CuO}$, and $<20 \mathrm{~nm}$ and $45 \mathrm{~nm} \mathrm{Al}_{2} \mathrm{O}_{3}$ respectively. A trend can be observed that as the concentration of nanoparticles within the base fluid increases both the thermal conductivity and viscosity also increase. It can be detrimental to a system with such high viscosity due to requiring an increasing amount of pumping power. While the thermal conductivity enhancement due to increased concentration can be appealing, the engineer or designer should be conscious of the also increasing viscosity. This increased viscosity can be mitigated though, by increasing the temperature of the nanofluid as seen through observation of Fig. 14 and 15, where the increasing temperature causes a decrease in viscosity.

In the case of thermal conductivity, two Brownian motion-based models are used to predict the experimental data gathered of the $38.4 \mathrm{~nm} \mathrm{Al}_{2} \mathrm{O}_{3}$ and 23.6nm CuO from Das [18] and Lee et al [11] respectively. The Patel et al [16] and Koo and Kleinstreur [10] models are able to be used due to the fact that the nanofluid compositions are within the validity range of each model. The models closely approximate the data with Patel et al [16] incurring and absolute average error of $0.68 \%$ for the $23.6 \mathrm{~nm} \mathrm{CuO}-$ Water nanofluid. While the Koo and Kleinstreur [10] model had an absolute average error of $0.37 \%$ with respect to the $38.4 \mathrm{~nm} \mathrm{Al} \mathrm{O}_{3}$-Water nanofluid presented by Lee et al [11]. Similarly, the viscosity of the $<20 \mathrm{~nm} \mathrm{Al}_{2} \mathrm{O}_{3}$ and $45 \mathrm{~nm} \mathrm{Al}_{2} \mathrm{O}_{3}$ presented by Pastorgoriza-Gallego [29] are predicted by the Rea [26] and Nguyen et al [25] models. The models are in close agreement with the experimental data with errors of $2.49 \%$ for Rea [26] in predicting the < 20nm nanofluid and $1.28 \%$ for Nguyen et al [25] approximating the $45 \mathrm{~nm} \mathrm{Al}_{2} \mathrm{O}_{3}$ nanofluid. 
TFEC-2020-32505

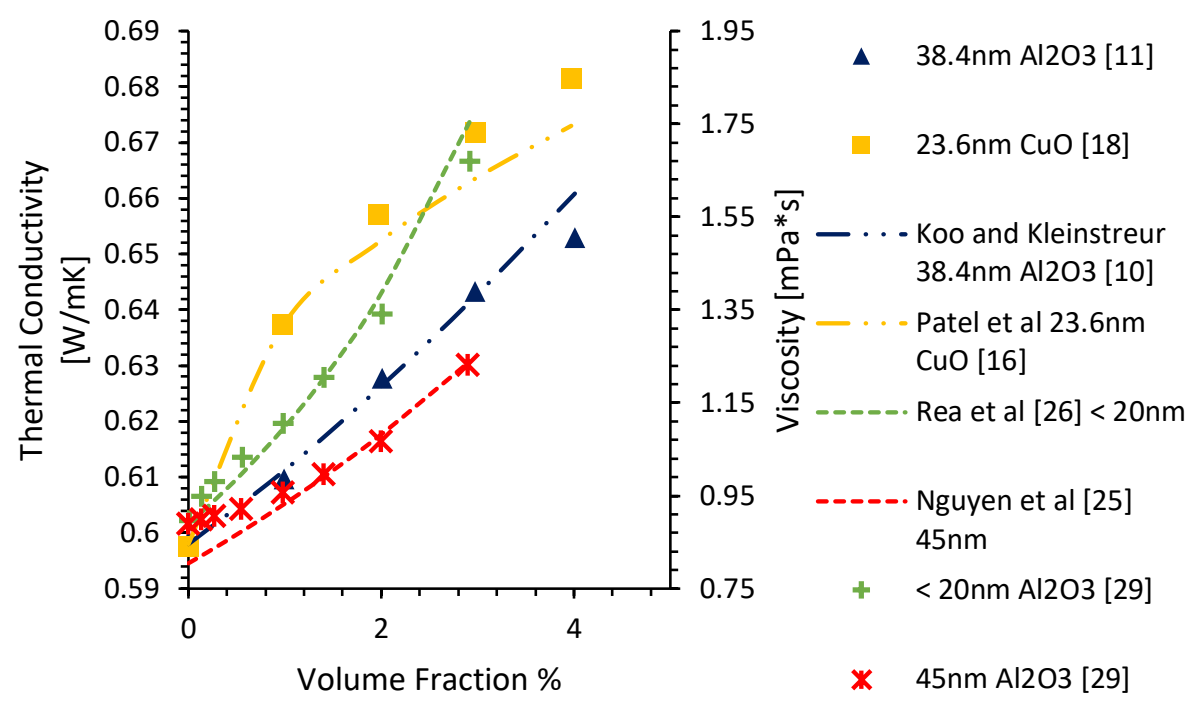

Fig. 13 Combined figure showing nanofluid thermal conductivity and viscosity dependency on volume fraction $\%$ of $38.4 \mathrm{~nm}[11],<20 \mathrm{~nm}$ and $45 \mathrm{~nm} \mathrm{Al}_{2} \mathrm{O}_{3}$ as well as 23.6nm [18] CuO. All with water as base fluid.

Shown below in Fig.14 is a combined graph of experimental data and theoretical models for both thermal conductivity and viscosity in order to observe the relationship for each property with respect to temperature. A common trend when increasing the temperature of a nanofluid is further enhancement in the thermal conductivity as can be seen in Fig. 6, 9 and Fig. 10-13. Fig. 13 shows data taken from Chon et al [15] and Nguyen et al [25]. The experimental data taken from Chon et al [15] shows the impact temperature has on the enhancement of thermal conductivity of the nanofluid. The nanofluid used was a $150 \mathrm{~nm} \mathrm{Al} \mathrm{O}_{3}$ nanoparticle at a $1 \%$ concentration by volume, within a de-ionized water base fluid. The trend can be observed in Fig. 14, as the temperature increases from $290 \mathrm{~K}-350 \mathrm{~K}$ the thermal conductivity consequently increases as well. Theoretical models shown such as Corcione [17], Vajiha and Das [14], Chon et al [15] and Patel et al [16] closely follow the same positive sloped trend with the increasing temperature. Through the characterization and consideration of the Brownian motion of particles within the nanofluid, these theoretical Brownian models show accurate predictions. With average percent errors of $8 \%$ or less compared to the experimental data [15].

In the case of viscosity of the nanofluid, as the temperature increases the viscosity tends to decrease. This inversely proportionate trend is shown in the same Fig.14. Here data is taken from Nguyen et al [25] of $47 \mathrm{~nm}_{\mathrm{Al}_{2} \mathrm{O}_{3}}$ nanoparticles with a volume concentration of $1 \%$ suspended in de-ionized water. The experimental data shows the decreasing nature of viscosity of the nanofluid as the temperature raises. Theoretical Models, Nguyen et al [25], Abu-Nada [28] and Khanafer and Vafai [27] follow this trend closely. Providing predictions that are in agreement with the experimental data provided. Absolute average of errors of the models shown relative to the experimental data are below 5\%. Nguyen et al [25], Abu-Nada [28] and Khanafer and Vafai [27] incur absolute average errors of $3.17 \%, 4.47 \%$ and $2.10 \%$ respectively.

It is important to consider the effects of thermal conductivity as well as viscosity when using nanofluids for applications in any field. In electronics for example it is crucial to have the capability to remove enough heat while also being able to properly pump the fluid. The enhancement in thermal conductivity of nanofluids is a major attraction to researchers but application is limited due to the increased viscosity (at room temperature) and pumping power required to pump fluid through a given system. It is shown through experimental data and theoretical correlations that as the temperature is increased the thermal conductivity enhancement is very apparent while the nanofluid becomes less viscous and thus easier to pump. Potential applications of nanofluids such as those given by Chon et al [15] and Nguyen et al [25] are electronics where the operating temperature is between 
$310 \mathrm{~K}-345 \mathrm{~K}(37 \mathrm{C}-72 \mathrm{C})$ where the nanofluids' thermal conductivity is significantly enhanced and viscosity significantly drops relative to at room temperature.

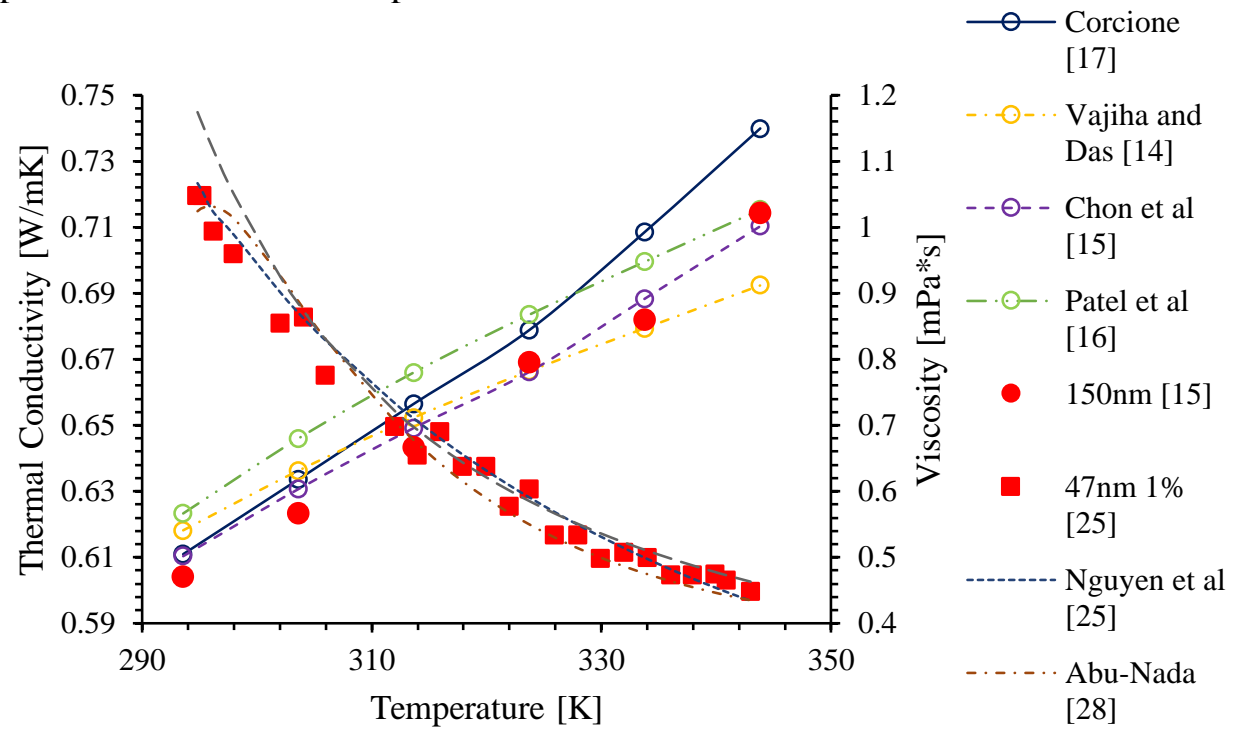

Fig. 14 Combined figure showing nanofluid thermal conductivity and viscosity dependency on temperature of $150 \mathrm{~nm}$ and $47 \mathrm{~nm} \mathrm{Al}_{2} \mathrm{O}_{3}$-Water nanofluids $[15,25]$.

Data acquired from Das [18] of $38.4 \mathrm{~nm} \mathrm{Al}_{2} \mathrm{O}_{3}$-Water nanofluids at concentrations of $1 \%$ and $4 \%$ as well as data from Nguyen et al [25] of $47 \mathrm{~nm} \mathrm{Al}_{2} \mathrm{O}_{3}$-Water nanofluids at concentrations of $1 \%$ and $7 \%$ help us observe trends due to both concentration and temperature shown in Fig. 15. Previously in Fig. 13 the downside to increased concentration was the increasing dynamic viscosity of the fluid. Fig. 15 shows that while at room temperature the viscosity of the nanofluid consisting of the $47 \mathrm{~nm} \mathrm{Al} \mathrm{O}_{3}$ with $7 \%$ concentration is much higher than that of the $1 \%$ concentration. But when increasing temperature, the viscosity begins to drop near to that of the $1 \%$ concentrated fluid. This shows the potential mitigation between increasing concentration for enhanced thermal conductivity while also increasing the viscosity, but when subjected to higher temperature reducing the viscosity of the nanofluid while retaining the enhanced thermal conductivity.

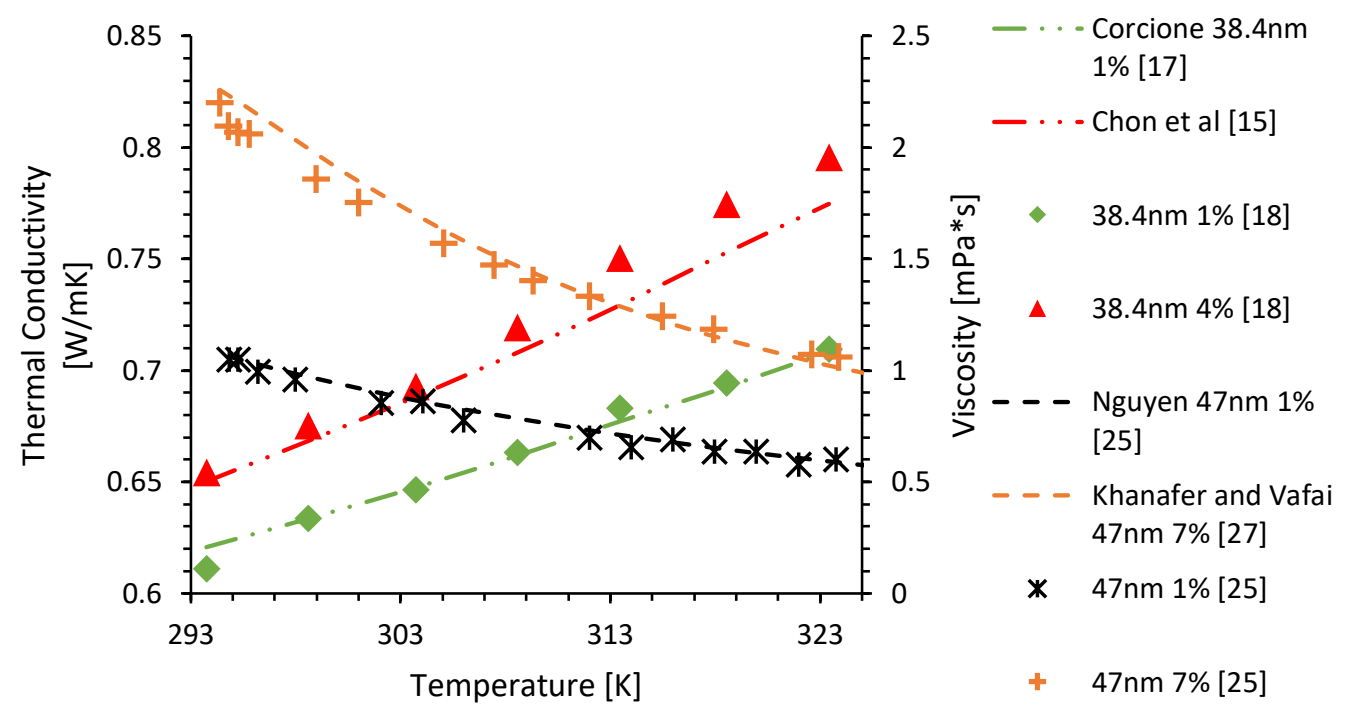

Fig. 15 Combined figure of $38.4 \mathrm{~nm} \mathrm{Al}_{2} \mathrm{O}_{3}-$ Water nanofluid at concentrations of $1 \%$ and $4 \%$ [18]. 47nm $\mathrm{Al}_{2} \mathrm{O}_{3}-$ Water nanofluid at concentrations of $1 \%$ and $7 \%$ [25] with varying temperature. 
Table 4 Theoretical expressions for thermal conductivity with remarks and assumptions

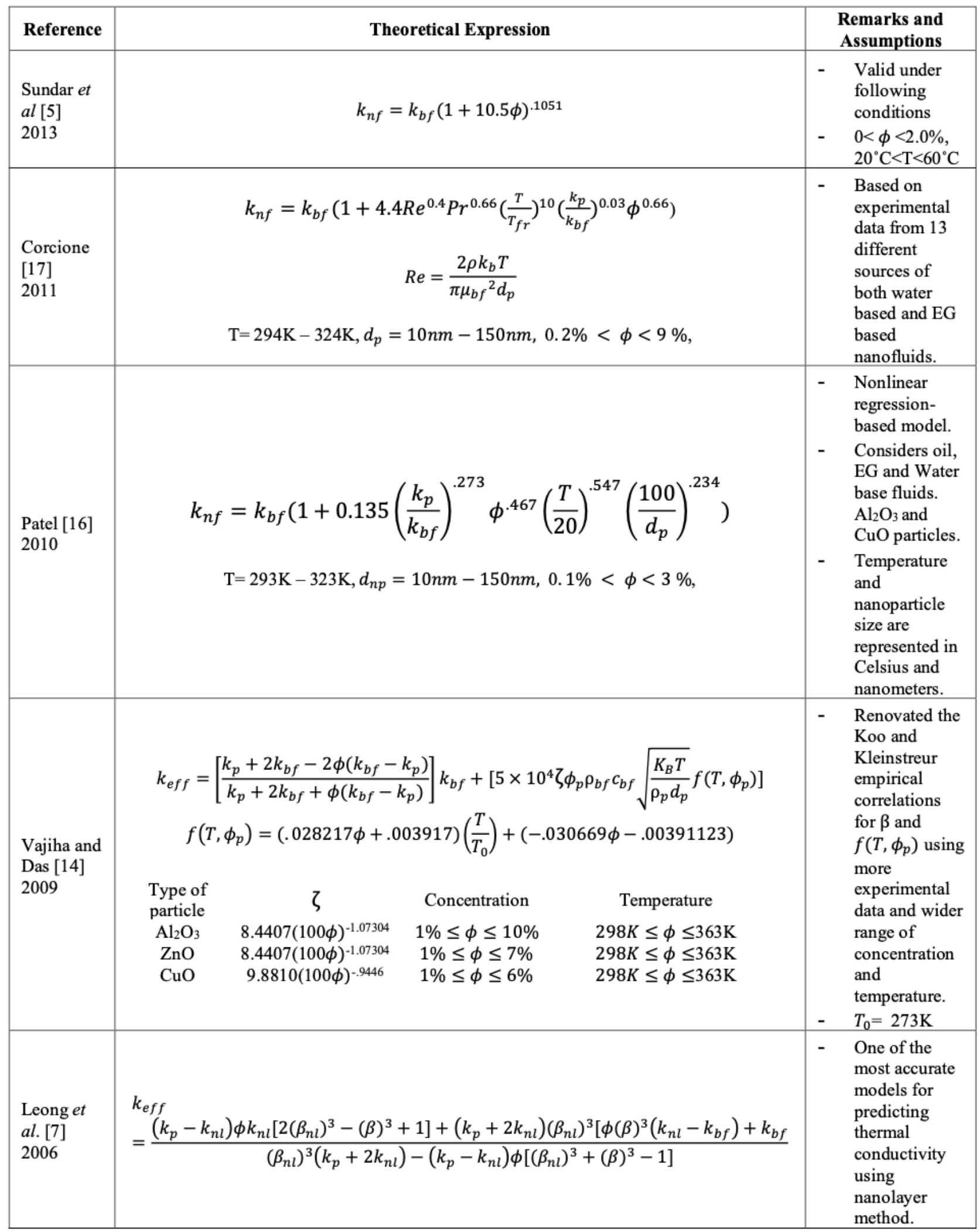




\begin{tabular}{|c|c|c|}
\hline Reference & Theoretical Expression & $\begin{array}{l}\text { Remarks and } \\
\text { Assumptions }\end{array}$ \\
\hline $\begin{array}{l}\text { Chon et al } \\
\text { [15] } 2005\end{array}$ & $\begin{array}{c}k_{e f f}=\left(1+64.7 \phi^{.746}\left(\frac{d_{b f}}{d_{n p}}\right)^{.369}\left(\frac{k_{n p}}{k_{b f}}\right)^{.746} \operatorname{Pr}^{.9955} \operatorname{Re}^{1.2321}\right) k_{b f} \\
\operatorname{Re}=\frac{\rho k_{b} T}{3 \pi \mu_{b f}{ }^{2} l_{b f}}\end{array}$ & 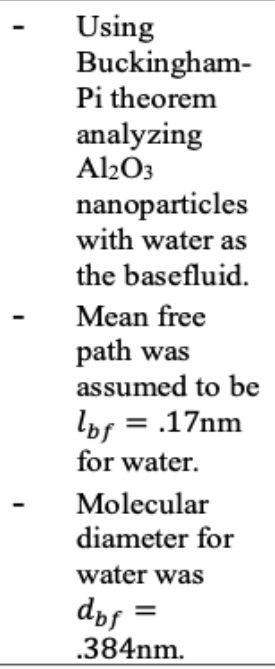 \\
\hline $\begin{array}{l}\text { Prasher et } \\
\text { al. }[9] \\
2005\end{array}$ & $\begin{array}{c}k_{e f f}=\left(1+A \operatorname{Re}^{M} \operatorname{Pr}^{0.333} \phi\right)\left[\frac{k_{p}+2 k_{b f}+2\left(k_{p}-k_{b f}\right) \phi}{k_{p}+2 k_{b f}-\left(k_{p}-k_{b f}\right) \phi}\right] k_{b f} \\
\alpha=\frac{2 R_{b} k_{m}}{d_{n p}} ; k_{m}=k_{b f}[1+.25 \operatorname{Re} * \operatorname{Pr}] ; \operatorname{Re}=\frac{1}{v_{b f}} \sqrt{\frac{18 k_{b f} T}{\pi \rho_{n p} d_{n p}}}\end{array}$ & 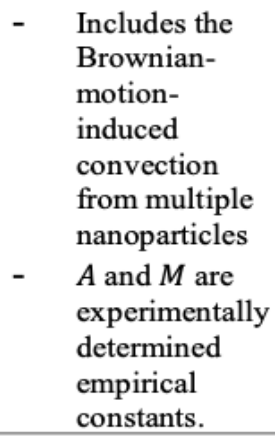 \\
\hline $\begin{array}{l}\text { Xie et al. } \\
{[6]} \\
2005\end{array}$ & $\begin{array}{c}\frac{k_{e f f}-k_{b f}}{k_{b f}}=3 \Theta \phi_{e f f}+\frac{3 \Theta^{2} \phi_{e f f}^{2}}{1-\Theta \phi_{e f f}} \\
\Theta=\frac{\left(\frac{k_{n l}-k_{b f}}{k_{n l}+2 k_{b f}}\right)\left[\left(1+\frac{\delta_{n l}}{r_{p}}\right)^{3}-\left[\frac{\left(k_{p}-k_{n l}\right)\left(k_{b f}+2 k_{n l}\right)}{\left(k_{p}+2 k_{n l}\right)\left(k_{b f}-k_{n l}\right)}\right]\right]}{\left(1+\frac{\delta_{n l}}{r_{p}}\right)^{3}+2\left[\left(\frac{k_{n l}-k_{b f}}{k_{n l}+2 k_{b f}}\right)\left(\frac{k_{p}-k_{n l}}{k_{p}+2 k_{n l}}\right)\right]}\end{array}$ & $\begin{array}{l}\text { The impact of } \\
\text { nanolayering } \\
\text { is taken into } \\
\text { account } \\
\text { through the } \\
\text { nanolayer } \\
\text { conductivity } \\
\text { and size. }\end{array}$ \\
\hline $\begin{array}{l}\text { Koo and } \\
\text { Kleinstreur } \\
{[10] 2004}\end{array}$ & 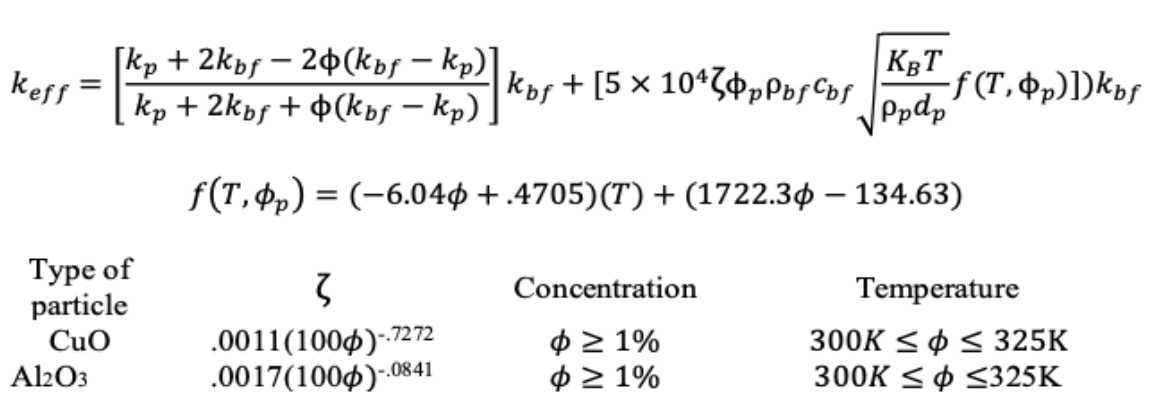 & $\begin{array}{l}\text { - Indicated that } \\
\text { Brownian } \\
\text { motion } \\
\text { produces } \\
\text { micromixing, } \\
\text { which in tur } \\
\text { makes it } \\
\text { dominant in } \\
\text { thermal } \\
\text { conductivity } \\
\text { enhancement. }\end{array}$ \\
\hline $\begin{array}{l}\text { Yu and } \\
\text { Choi }[8] \\
2003\end{array}$ & $k_{e f f}=\frac{k_{p}+2 k_{b f}+2\left(k_{p}-k_{b f}\right)(1-\gamma)^{3} \phi}{k_{p}+2 k_{b f}-\left(k_{p}-k_{b f}\right)(1+\gamma)^{3} \phi} k_{b f}$ & $\begin{array}{l}\text { Nanolayer } \\
\text { model taking } \\
\text { into account } \\
\text { the nanolayer } \\
\text { thickness as } \\
\text { well as the } \\
\text { size of the } \\
\text { nanoparticle }\end{array}$ \\
\hline
\end{tabular}


TFEC-2020-32505

\begin{tabular}{|c|c|c|}
\hline Reference & Theoretical Expression & $\begin{array}{l}\text { Remarks and } \\
\text { Assumptions }\end{array}$ \\
\hline $\begin{array}{l}\text { Hamilton- } \\
\text { Crosser [4] } \\
1962\end{array}$ & $k_{n f}=k_{b f}\left[\frac{k_{n f}+(n-1) k_{b f}-(n-1) \phi\left(k_{b f}-k_{n f}\right)}{k_{n f}+(n-1) k_{b f}+\phi\left(k_{b f}-k_{n f}\right)}\right]$ & $\begin{array}{l}\text { - Use } \mathrm{n}=3 \text { for } \\
\text { spherical and } \\
\mathrm{n}=6 \text { for } \\
\text { cylindrical } \\
\text { shaped } \\
\text { particles }\end{array}$ \\
\hline $\begin{array}{l}\text { Maxwell } \\
{[2]} \\
1904\end{array}$ & $k_{n f}=\frac{k_{p}+2 k_{b f}+2 \phi\left(k_{p}-k_{b f}\right)}{k_{p}+2 k_{b f}-\phi\left(k_{p}-k_{b f}\right)} k_{b f}$ & $\begin{array}{ll}\text { - } & \text { Valid for } \\
\text { Spherical } \\
\text { particles } \\
\text { - } & \text { Static and } \\
\text { homogenous } \\
\text { mixing }\end{array}$ \\
\hline
\end{tabular}


Table 5 Theoretical expressions for viscosity with remarks and assumptions

\begin{tabular}{|c|c|c|}
\hline Reference & Theoretical Expression & $\begin{array}{l}\text { Remarks and } \\
\text { Assumptions }\end{array}$ \\
\hline $\begin{array}{l}\text { Khanafer and } \\
\text { Vafai [27] } \\
2011\end{array}$ & $\begin{aligned} \mu_{n f}=-0.4491+ & \frac{28.4312}{T}+0.574 \phi-0.1634 \phi^{2}+\frac{23.053 \phi^{2}}{T^{2}}+0.0132 \phi^{3} \\
& -\frac{2354.735 \phi}{T^{3}}+\frac{23.498 \phi^{2}}{d_{p}^{2}}-\frac{3.0185 \phi^{3}}{d_{p}^{2}}\end{aligned}$ & $\begin{array}{ll}- & \text { Data from Anoop et } \\
\text { al [31], Nguyen } \text { et al } \\
{[25], \text { Pak and Cho }} \\
{[29], \text { and Putra } \text { et al }} \\
{[30]} \\
\text { - } \\
\text { Particle Diameter } \\
\text { range from } 13 \mathrm{~nm}- \\
131 \mathrm{~nm} \\
-\quad \begin{array}{l}\text { Volume fraction } \\
\text { range from } 1-9 \%\end{array} \\
-\quad \text { Temperature range } \\
\text { from } 20-70^{\circ} \mathrm{C}\end{array}$ \\
\hline $\begin{array}{l}\text { Abu-Nada [28] } \\
2011\end{array}$ & $\begin{array}{c}\mu_{n f}=-0.155-\frac{19.582}{T}+0.794 \phi \frac{+2094.47}{T^{2}}-0.192 \phi^{2}-8.11 \frac{\phi}{T}-\frac{27463.863}{T^{3}} \\
+0.0127 \phi^{3}+1.6044 \frac{\phi^{2}}{T}+2.175 \frac{\phi}{T^{2}}\end{array}$ & $\begin{array}{l}\text { - } \begin{array}{l}\text { Developed model } \\
\text { based off Nguyen } \text { et } \\
\text { al's [61] data for }\end{array} \\
\mathrm{Al}_{2} \mathrm{O}_{3} \text { nanofluids } \\
\text { - } \quad \begin{array}{l}\text { Considered two- } \\
\text { dimensional }\end{array} \\
\text { regression analyses } \\
\text { as a function of } \\
\text { volume fraction and } \\
\text { temperature }\end{array}$ \\
\hline $\begin{array}{l}\text { Rea et al [30] } \\
2008\end{array}$ & $\begin{array}{c}\frac{\mu_{n f}}{\mu_{b f}}=e^{(4.91 \phi / 0.2092-\phi)} \\
\mu_{n f}=\mu_{b f}\left(1+46.801 \phi+550.82 \phi^{2}\right)\end{array}$ & 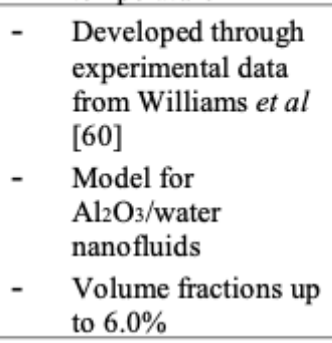 \\
\hline $\begin{array}{l}\text { Nguyen et al } \\
{[25]} \\
2007\end{array}$ & $\begin{array}{c}\mu_{n_{f}}=\mu_{f}\left(1+0.025 \phi+0.015 \phi^{2}\right) \\
36 \mathrm{~nm} \mathrm{Al}_{2} \mathrm{O}_{3} \\
\frac{\mu_{n f}}{\mu_{b f}}=0.904 e^{0.148 \phi} \\
47 \mathrm{~nm} \mathrm{Al}_{2} \mathrm{O}_{3}\end{array}$ & $\begin{array}{ll}- & \begin{array}{l}\text { Developed through } \\
\text { experimental data }\end{array} \\
\text { - } & \text { Models for } \\
\mathrm{Al}_{2} \mathrm{O}_{3} / \text { water } \\
\text { nanofluid } \\
-\quad \text { Particle sizes of } \\
\text { 36nm and } 47 \mathrm{~nm} \\
\text { shown respectively }\end{array}$ \\
\hline $\begin{array}{l}\text { Nguyen et al } \\
{[25]} \\
2007\end{array}$ & $\mu_{n f}=\mu_{f}\left(1.475-0.319 \phi+0.051 \phi^{2}+0.009 \phi^{3}\right)$ & $\begin{array}{ll} & \begin{array}{l}\text { Created model from } \\
\text { experimentation }\end{array} \\
- & \begin{array}{l}\text { For } 29 \mathrm{~nm} \mathrm{CuO} \\
\text { nanofluids }\end{array} \\
\end{array}$ \\
\hline $\begin{array}{l}\text { Maïga et al } \\
{[24]} \\
2004\end{array}$ & $\mu_{n f}=\mu_{f}\left(1+10.6 \phi+(10.6 \phi)^{2}\right)$ & $\begin{array}{l}\text { Viscosity correlation } \\
\text { through regression } \\
\text { analysis } \\
\text { Experimental data for } \\
\mathrm{TiO}_{2} \text { nanofluids }\end{array}$ \\
\hline $\begin{array}{l}\text { Lundgren [23] } \\
1972\end{array}$ & $\mu_{n f}=\mu_{b f}\left(1+2.5 \varphi+\frac{25}{4} \varphi^{2}\right)$ & $\begin{array}{l}\text { Taylor series } \\
\text { extension of } \\
\text { Einstein's original } \\
\text { law of viscosity. }\end{array}$ \\
\hline $\begin{array}{l}\text { Brinkman [22] } \\
1952\end{array}$ & $\mu_{n f}=\mu_{b f}\left(\frac{1}{(1-\varphi)^{2,3}}\right)$ & $\begin{array}{ll}\text { - } & \text { Extended Einstein } \\
& \text { equation for use with }\end{array}$ \\
\hline
\end{tabular}




\begin{tabular}{|c|c|c|}
\hline Reference & Theoretical Expression & $\begin{array}{l}\text { Remarks and } \\
\text { Assumptions }\end{array}$ \\
\hline & & $\begin{array}{l}\text { moderate particle } \\
\text { concentrations. }\end{array}$ \\
\hline $\begin{array}{l}\text { Einstein [21] } \\
1905\end{array}$ & $\mu_{n f}=1+2.5 \varphi$ & $\begin{array}{ll}\text { - } & \text { Predicts effective } \\
\text { viscosity of fluid. } \\
\text { - } & \text { Applicable to limited } \\
\text { low volume } \\
\text { concentrations }(\varphi< \\
.02 \%) .\end{array}$ \\
\hline
\end{tabular}

\section{CONCLUSIONS}

By considering different characteristics of the nanofluid including but not limited to: nanoparticle material, volume fraction, particle shape, nanolayer and Brownian motion the error in determining the thermal conductivity of the nanofluid can effectively be reduced. It can be difficult to obtain all the characteristics of the nanofluid such as the nanolayer thickness and thermal conductivity of the nanolayer but the more information on the characteristics and interactions can help to increase the accuracy of the approximations. In the case of Effective Medium Theory based formulas for calculating thermal conductivity, the Hamilton Crosser [3] model shows relatively low average error percentages of $<15 \%$ when applied to the 10 experimental data sets shown in Table 1 . While the Maxwell [2] and Wasp [4] equations tend to incur greater errors. Another observable trend relating to the EMT models discussed in this paper, are that as concentration increases the models tend to underestimate the experimental thermal conductivities.

If nanolayer characteristics can be determined the Leong et al [7] model could be applied with the assumption of $k_{n l}=2 k_{b f}$. This model had the least amount of error within the presented data sets shown in Table 2, compared to other existing nanolayer methods. The Leong et al [7] model had an average error percentage of $4.69 \%$ compared to $6.09 \%$ and $6.81 \%$ for Yu and Choi [8] and Xie [6] et al models respectively.

When considering the thermal conductivity of the nanofluid as a function of varying temperature, Brownian movement-based equations could be utilized for the relatively accurate results. Patel et al [16] seems to best approximate the $\mathrm{Al}_{2} \mathrm{O}_{3}$-water nanofluid data presented in Table 3 with an absolute average error of $2.54 \%$ across all experimental data gathered for the fluid. The Chon et al [15] model similarly predicts the thermal conductivity of the $\mathrm{Al}_{2} \mathrm{O}_{3}$ nanofluids with an average error of $3.15 \%$ from the same data. Koo and Kleinstreur [10] best predicted the thermal conductivity of the $\mathrm{CuO}$-water nanofluid experimental data, closely followed by Vajiha and Das [14] with absolute average errors of $3.24 \%$ and $3.51 \%$ respectively.

Other observations not regarding to accuracy of models have been noted throughout the paper. The most significant being throughout Fig. 13 - 15 in which the thermal conductivity and viscosity are both considered. In the case of volume fraction concentration, the observation made was that as the concentration increases both the viscosity and thermal conductivity both increases. The downside here being the increased viscosity making the fluid more difficult to pump throughout a system. From observations the way to mitigate this increased viscosity is to increase the temperature of the fluid causing an inverse relationship between temperature and viscosity. Thus, preserving the enhancement in thermal conductivity while effectively reducing viscosity at temperature of $315 \mathrm{~K}$ and higher. Making nanofluids with such characteristics of viscosity and thermal conductivity potentially applicable to cooling electronics which have operating temperatures of $60 \mathrm{C}-80 \mathrm{C}$. With respect to thermal conductivity other factors such as size were also observed. From the data gathered one can conclude that particles of smaller sizes will outperform larger particles in enhancing the thermal conductivities of the nanofluid with respect to the base fluid. 


\section{NOMENCLATURE}

\begin{tabular}{|c|c|c|c|c|c|}
\hline$k$ & $\begin{array}{l}\text { Thermal } \\
\text { conductivity of } \\
\text { nanofluid }\end{array}$ & $(\mathrm{W} / \mathrm{mK})$ & $\phi$ & $\begin{array}{l}\text { Volume fraction of } \\
\text { nanoparticle in base } \\
\text { fluid }\end{array}$ & $(-)$ \\
\hline$v$ & Kinematic viscosity & $\left(\mathrm{m}_{2} / \mathrm{s}\right)$ & $n$ & Empirical shape factor & $(-)$ \\
\hline$k_{b}$ & Boltzmann Constant & $(-)$ & $\delta n l$ & Nanolayer thickness & $(-)$ \\
\hline $\mathrm{T}$ & Temperature & (K) & $\beta_{n l}$ & $\begin{array}{l}\text { Dimensionless } \\
\text { nanolayer parameter }\end{array}$ & $(-)$ \\
\hline A & Empirical constant & $(-)$ & $\beta$ & Empirical correlation & $(-)$ \\
\hline $\operatorname{Re}$ & Reynolds number & $(-)$ & $\gamma$ & $\begin{array}{l}\text { ratio of nanolayer } \\
\text { thickness to } \\
\text { nanoparticle radius }\end{array}$ & $(-)$ \\
\hline $\operatorname{Pr}$ & $\begin{array}{l}\text { Prandtl number of } \\
\text { base fluid }\end{array}$ & $(-)$ & $\rho$ & Density & $\left(\mathrm{kg} / \mathrm{m}_{3}\right)$ \\
\hline$M$ & Empirical constant & $(-)$ & $\mathrm{Bi}$ & Biot number & $(-)$ \\
\hline $\mathrm{T}$ & Temperature & $(\mathrm{K})$ & $\zeta$ & Empirical constant & $(-)$ \\
\hline $\mathrm{T}_{0}$ & $\begin{array}{l}\text { Reference } \\
\text { temperature }\end{array}$ & (K) & $\alpha$ & Thermal diffusivity & {$\left[\mathrm{m}_{2} / \mathrm{s}\right]$} \\
\hline$c_{p}$ & Specific heat & $(\mathrm{J} / \mathrm{kgK})$ & $\mathrm{d}$ & Diameter & (m) \\
\hline $\mathrm{Rb}$ & $\begin{array}{l}\text { Thermal boundary } \\
\text { resistance }\end{array}$ & $(\mathrm{m} 2 \mathrm{~K} / \mathrm{W})$ & $\mu$ & Dynamic viscosity & $(\mathrm{mPa} * \mathrm{~s})$ \\
\hline$r$ & Radius & (m) & & & \\
\hline \multicolumn{6}{|l|}{ Subscripts } \\
\hline & Particle & & & & \\
\hline$b f$ & Base fluid & & & & \\
\hline$m$ & Matrix & & & & \\
\hline & Nanofluid & & & & \\
\hline
\end{tabular}

\section{REFERENCES}

[1] Saidur R., Leong K. Y., and Mohammad H. A.. "A Review on Applications and Challenges of Nanofluids," Renewable and Sustainable Energy Reviews, 15.3, pp. 1646-668, (2011):

[2] Maxwell J. C., "A Treatise on Electricity and Magnetism," Claredon, Oxford, UK, (1891).

[3] Hamilton R. L, Crosser O. K., "Conductivity of Heterogeneous Two-Component Systems," I \& EC Fundamentals, vol. 1, pp. 182-191, (1962).

[4] Wasp F.J, "Solid-Liquid Slurry Pipeline Transportation”, Trans., Tech, Berlin, (1977)

[5] Sundar L. S., "Investigation of Thermal Conductivity and Viscosity of $\mathrm{Fe}_{3} \mathrm{O}_{4}$ nanofluid for heat transfer applications." Int. Comm. in Heat and Mass Transf., vol. 44, pp. 1-8, (2013).

[6] Xie H., Fujii M., Zhang X., "Effect of Interfacial Nanolayer on the Effective Thermal Conductivity of Nanoparticle-Fluid Mixture”, Int. J. Heat Mass Transf., vol. 48, pp. 2926-2932, (2005).

[7] Leong K. C., Murshed S. M. S., "A model for the Thermal Conductivity of Nanofluids The Effect of Interfacial Layer", J. of Nanoparticle Research., vol. 8, pp. 245-254, (2006).

[8] Yu W., and Choi S. U. S., "The Role of Interfacial Layers in the Enhanced Thermal Conductivity of Nanofluids: A Renovated Maxwell Model," J. of Nanoparticle Research., vol. 5, pp. 167-171, (2003).

[9] Prasher R., Bhattacharya P., Phelan P. E., "Brownian-Motion-Based-Convective-Conductive Model for Effective Thermal Conductivity of Nanofluids," ASME Trans. J. Heat Transf., vol. 128, pp. 588-595, (2006).

[10] Koo J., Kleinstreuer C. A., "New Thermal Conductivity Model for Nanofluids," J. of Nanoparticle Research., vol. 6, no. 6, pp. 577-588, (2004).

[11] Lee S., Choi U. S., and Eastman J. A., "Measuring Thermal Conductivity of Fluids Containing Oxide Nano Particles," ASME Trans. J. Heat Transf., vol. 121, pp. 280-289, (1999).

[12] Vajpai A. and Rajvanshi A. K., Thermal Conductivity of Nanofluids Experimental Investigation of Thermal Conductivity Enhancement with Nanofluids, LAMBERT Academic Publishing, pp. 30-35, (2012).

[13] Tinga W.R., Voss W.A.G and Blossey D.F., "Generalized approach to multiphase dielectric mixture theory," J. Appl. Phys. pp. 3897-3903, (1973). 
[14] Vajiha R.S., Das D.K., "Experimental Determination of Thermal Conductivity of Three Nanofluids and Development of New Correlations," ASME J. of Heat and Mass Transf., vol 52, pp. 4675-4682., (2009).

[15] Chon C.H., Kihm K.D., Lee S.P. and Choi S.U.S "Empirical Correlation Finding the Role of Temperature and Particle Size for Nanofluid $\left(\mathrm{Al}_{2} \mathrm{O}_{3}\right)$ Thermal Conductivity Enhancement," Appl. Phys. Lett. 87, 153107 (2005).

[16] Patel E. H., Sundararajan T., Das K. S. "An experimental investigation into the thermal conductivity enhancement in oxide and metallic nanofluids,” J. Nanopart. Res. vol. 12, pp.1015-1031 (2007).

[17] Corcione M., "Empirical correlating equations for predicting the effective thermal conductivity and dynamic viscosity of nanofluids," Energy Conserv. And Mgmt.., vol. 52, pp.789-793 (2011).

[18] Das K.S., Putra N., Thiesen P., Roetzel W., "Temperature Dependence of Thermal Conductivity Enhancement for Nanofluids" Trans of the ASME, vol.125, pp.567-577 (2003).

[19] Masuda H., Ebata A., Teramae K., Hishinuma N., "Alteration of Thermal Conductivity and Viscosity of Liquid by Dispersing Ultra-fine Particles". pp. 227-233., (1993)

[20] Eastman J.A., Phillpot S. R., Choi S.U.S., Keblinski P., “Thermal Transport in Nanofluids” Annu. Rev. Mater. Res. Vol. 34, pp. 219-246. (2004)

[21] Einstein A., "Investigations on the theory of the Brownian Movement", (1905)

[22] Brinkman C., "The Viscosity of Concentrated Suspensions and Solutions" The J. of Chem. Phys. vol. 20, pp. 571. (1952)

[23] Lundgren S., "Slow Flow Through Stationary Random Beds and Suspensions" J. Fluid Mech. vol. 52, pp. 273-299. (1971)

[24] Maïga B. S., Nguyen C. T., Galanis N. Roy G., "Heat Transfer Behaviours of Nanofluids in a Uniformly Heated Tube" Superlattices and Microstr. vol. 35. pp. 543-557 (2004)

[25] Nguyen C.T., Desgranges F., Roy G., Galanis N., Maré T., Boucher S., Angue Mintsa H., "Temperature and particle-sized dependent viscosity data for water-based nanofluids - Hysteresis phenomenon," Int. J. Heat Fluid Flow, 28, pp. 1492-1506, (2007).

[26] Rea R., McKrell T., Hu L., Buongiorno J., "Laminar Convective Heat Transfer and Viscous Pressure Loss of Alumina-Water and Zirconia-Water nanofluids", Int. J. Heat and Mass Transf., vol. 52, pp. 2042-2048. (2009)

[27] Khanafer K., Vafai K., "A critical synthesis of thermophysical characteristics of nanofluids", Int. J. Heat. Mass. Transf. vol. 55, pp. 4410-4428, (2011).

[28] Abu-Nada E., "Rayleigh-Bénard convection in nanofluids: Effect of temperature dependent properties", Int. J. Therm. Sci., vol. 50, pp. 1720-1730, (2011).

[29] Pastoriza-Gallego M. J., Casanova C., Páramo R., Barbés B., Legido J. L., Piñeiro M. M., "A study on stability and thermophysical properties (density and viscosity) of $\mathrm{Al}_{2} \mathrm{O}_{3}$ in water nanofluid”, J. Appl. Phys., 106, 064301, pp. 1-8, (2009).

[30] Pak B.C., Cho Y.I., "Hydrodynamic and Heat Transfer Study of Dispersed Fluids with Submicron Metallic Oxide Particles", Exp. Heat Transfer, vol. 11, pp.. 151-170. (1999)

[31] Putra N., Roetzel., Das S.K., "Natural Convection of Nanofluids", Heat Mass Transf., vol.39, pp. 775-784. (2003)

[32] Anoop K.B., Kabelac S., Sundararajan T., Das S.K., "Rheological and Flow Characteristics of Nanofluids: Influence of Electroviscous Effects and Particle Agglomeration", J. Appl. Phys., vol. 106, (2009)

[33] Murshed S. M. S., Leong K.C., Yang C., "Thermophysical and Electrokinetic Properties of Nanofluids- A Critical Review". Appl. Therm. Eng., vol. 28, pp.2109-2125. (2008)

[34] Wang X., Xu X., Choi S.U.S., Thermophys. Heat Transf., vol. 13, pp. 474. (1999) 